\title{
Proinflammatory NFkB signalling promotes mitochondrial dysfunction in skeletal muscle in response to cellular fuel overloading
}

\author{
Raid B. Nisr ${ }^{1} \cdot$ Dinesh S. Shah ${ }^{1} \cdot \operatorname{lan}$ G. Ganley ${ }^{2} \cdot$ Harinder S. Hundal ${ }^{1}$ \\ Received: 28 January 2019 / Revised: 8 May 2019 / Accepted: 13 May 2019 / Published online: 17 May 2019 \\ (C) The Author(s) 2019
}

\begin{abstract}
Sustained nutrient (fuel) excess, as occurs during obesity and diabetes, has been linked to increased inflammation, impaired mitochondrial homeostasis, lipotoxicity, and insulin resistance in skeletal muscle. Precisely how mitochondrial dysfunction is initiated and whether it contributes to insulin resistance in this tissue remains a poorly resolved issue. Herein, we examine the contribution that an increase in proinflammatory NFkB signalling makes towards regulation of mitochondrial bioenergetics, morphology, and dynamics and its impact upon insulin action in skeletal muscle cells subject to chronic fuel (glucose and palmitate) overloading. We show sustained nutrient excess of L6 myotubes promotes activation of the IKK $\beta$-NFkB pathway (as judged by a six-fold increase in IL-6 mRNA expression; an NFkB target gene) and that this was associated with a marked reduction in mitochondrial respiratory capacity $(>50 \%)$, a three-fold increase in mitochondrial fragmentation and 2.5 -fold increase in mitophagy. Under these circumstances, we also noted a reduction in the mRNA and protein abundance of PGC1 $\alpha$ and that of key mitochondrial components (SDHA, ANT-1, UCP3, and MFN2) as well as an increase in cellular ROS and impaired insulin action in myotubes. Strikingly, pharmacological or genetic repression of NFkB activity ameliorated disturbances in mitochondrial respiratory function/morphology, attenuated loss of SDHA, ANT-1, UCP3, and MFN2 and mitigated the increase in ROS and the associated reduction in myotube insulin sensitivity. Our findings indicate that sustained oversupply of metabolic fuel to skeletal muscle cells induces heightened NFkB signalling and that this serves as a critical driver for disturbances in mitochondrial function and morphology, redox status, and insulin signalling.
\end{abstract}

Keywords NFkB $\cdot$ Muscle $\cdot$ Glucose $\cdot$ Palmitate $\cdot$ Insulin $\cdot$ Mitochondria $\cdot$ Respiration $\cdot$ Metabolism

$\begin{array}{ll}\text { Abbreviations } \\ \text { GLC } & \text { Glucose } \\ \text { PA } & \text { Palmitate } \\ \text { FAs } & \text { Free fatty acids } \\ \text { DAG } & \text { Diacylglycerol } \\ \text { NFKB } & \begin{array}{l}\text { Nuclear factor kappa-light-chain-enhancer of } \\ \text { activated B cells }\end{array} \\ \text { IL6 } & \text { Interleukin-6 }\end{array}$

Electronic supplementary material The online version of this article (https://doi.org/10.1007/s00018-019-03148-8) contains supplementary material, which is available to authorized users.

Harinder S. Hundal

h.s.hundal@dundee.ac.uk

1 Division of Cell Signalling and Immunology, Sir James Black Centre, School of Life Sciences, University of Dundee, Dundee DD1 5EH, UK

2 MRC Protein Phosphorylation and Ubiquitylation Unit, Sir James Black Centre, School of Life Sciences, University of Dundee, Dundee DD1 5EH, UK

\begin{tabular}{|c|c|}
\hline $\begin{array}{l}\text { TNF } \\
\text { IKK } \beta\end{array}$ & $\begin{array}{l}\text { Tumor necrosis factor- } \alpha \\
\text { Inhibitor of nuclear factor kappa-B kinase subu- } \\
\text { nit beta }\end{array}$ \\
\hline $\mathrm{IKB} \alpha$ & Inhibitor of nuclear factor kappa-B \\
\hline ROS & Reactive oxygen species \\
\hline ANT-1 & Adenine nucleotide translocase type 1 \\
\hline UCP3 & Uncoupling protein 3 \\
\hline SDHA & Succinate Dehydrogenase \\
\hline PGC $1 \alpha$ & $\begin{array}{l}\text { Peroxisome proliferator-activated receptor } \\
\text { gamma coactivator } 1 \text {-alpha }\end{array}$ \\
\hline MitoQ & Mitoquinone \\
\hline TLR-4 & Toll-like receptor- 4 \\
\hline 2DG & 2-Deoxyglucose \\
\hline FCCP & $\begin{array}{l}\text { Carbonilcyanide } \\
p \text {-trifluromethoxyphenylhydrazone }\end{array}$ \\
\hline OPA1 & Optic atrophy 1 \\
\hline MFN2 & Mitofusin 2 \\
\hline DRP1 & Dynamin-related protein 1 \\
\hline
\end{tabular}




\section{Introduction}

There is mounting evidence that chronic activation of proinflammatory signalling in tissues such as skeletal muscle and adipose is a significant contributing factor in the development and progression of metabolic disorders such as insulin resistance, obesity and Type II diabetes $[1,2]$. This inflammatory response is triggered by circulating proinflammatory cytokines such as interleukin-6 (IL-6), tumour necrosis factor- $\alpha$ (TNF $\alpha$ ) and by sustained increases in the concentration of free fatty acids (FAs), such as palmitate. The actions of these stimuli serve to not only further induce tissue expression and secretion of IL-6 and TNF $\alpha$ (via the NFKB pathway, [3, 4]), but impair control of numerous signalling pathways regulating skeletal muscle insulin signalling, glucose uptake, mitochondrial fuel oxidation and respiration that impact negatively upon skeletal muscle energy homeostasis [2, 5-7]. Indeed, mitochondrial dysfunction and/or a reduction in mitochondrial content, as characterised by a fall in oxidative capacity, has been reported in skeletal muscle and adipose tissue from obese and diabetic subjects $[8$, 9]. Notably, the sustained oversupply of metabolic fuel (glucose and fatty acids) to skeletal muscle, as seen during Type II diabetes and obesity, impairs the ability of mitochondria to shift between use of lipid during fasting and use of carbohydrate in the post-prandial state. This metabolic inflexibility imposes a major substrate burden on the oxidative machinery of muscle and the continued oversupply of carbon fuel eventually surpasses the respiratory drive and cellular demand for ATP synthesis [10]. As a result, FAs undergo incomplete oxidation and greater partitioning into lipotoxic derivatives [e.g., diacylglycerol (DAG) and ceramides] which have been strongly implicated in the pathogenesis of insulin resistance $[11,12]$. Whilst some have suggested that sufficient mitochondrial capacity remains under such circumstances [13], others submit that without energy demand such capacity is irrelevant and that metabolic dysfunction associated with the "effective" mitochondrial insufficiency may exacerbate effects of lipotoxicity [14-18].

Mitochondrial fuel overload also increases production of reactive oxygen species (ROS) $[19,20]$ that may serve to trigger or prime mechanisms that not only induce, but also sustain the loss in mitochondrial function. For example, it is has been suggested that increased ROS generation may suppress expression of PGC $1 \alpha$ (a major regulator of mitochondrial biogenesis) and that of genes encoding components of the respiratory chain, but may also act as a stimulus activating proinflammatory NFkB signalling that intersects with processes influencing mitochondrial function [21, 22]. In support of this latter idea, signalling initiated by toll-like receptor-4 (TLR-4) and the tumor necrosis factor- $\alpha$ (TNF- $\alpha$ ) receptor results in activation of the NFkB pathway, which has been linked to reduced mitochondrial respiration and suppressed activation of transcriptional regulators that promote mitochondrial biogenesis and the shift towards a muscle oxidative phenotype [23, 24]. We, and others, have also previously demonstrated that obesity in rodents and chronic oversupply of metabolic fuel to skeletal muscle cells in vitro is associated with an increase in proinflammatory NFkB signalling and insulin resistance $[17,25,26]$. We hypothesise that sustained oversupply of metabolic fuel will promote activation of proinflammatory NFkB signalling in muscle cells and that this contributes significantly to disturbances in mitochondrial biology that impact negatively upon myocellular insulin sensitivity. The studies reported herein have tested this proposition.

\section{Materials and methods}

\section{Chemicals and reagents}

Culture media $\alpha$-MEM ( $\alpha$-minimum essential medium), DMEM (Dulbecco's Modified Eagle's medium), Medium 199, foetal bovine serum, horse serum, Mitosox, prolong diamond antifade mountant, and mitotracker dyes were all purchased from Thermo Fisher Scientific (UK). $\alpha$-MEM media-lacking glucose was purchased from PAN Biotech, UK, Mitoquinone (Mito Q) was obtained from Cambridge biosciences, UK). BI605906 was a generous gift from Professor Sir Philip Cohen (MRC Protein Phosphorylation Unit, University of Dundee), but also purchased from Tocris (Bristol, UK), MitoSpy ${ }^{\mathrm{TM}}$ Green FM was from BioLegends, UK and MitoPYI, Mitotempo, hepatocyte growth factor, dexamethasone, basic FGF, gelatine, vitamin B12, retinoic acid, VAS2870, palmitate, oligomycin, FCCP (carbonyl cyanide p-trifluoromethoxyphenylhydrazone), rotenone, antimycinA, hygromycin B, apo-transferrin human, $\mathrm{SYBR}^{\circledR}$ Green JumpStart Taq Ready Mix, and Polyberen were all purchased Sigma-Aldrich, UK).

\section{Rat and human skeletal muscle cell culture, transfection and fatty acid treatment}

L6 muscle cells were cultured to myotubes as described previously [27] in $\alpha$-minimal essential media ( $\alpha \mathrm{MEM}$ ) containing $2 \%(\mathrm{v} / \mathrm{v})$ foetal bovine serum (FBS) and 1\% (v/v) antibiotic/antimycotic solution (100 units/ml penicillin, $100 \mu \mathrm{g} / \mathrm{ml}$ streptomycin, and $250 \mathrm{ng} / \mathrm{ml}$ amphotericin B) at $37{ }^{\circ} \mathrm{C}$ with $5 \% \mathrm{CO}_{2}$. In some experiments, L6 myotubes were infected with adenovirus harbouring a mutated I $\mathrm{B} \alpha \mathrm{S} 32 \mathrm{~A} / \mathrm{S} 36 \mathrm{~A}$ construct. This virus was kindly provided 
by Dr Harry Heimberg (Vrije Universiteit Brussel, Belgium) and was initially propagated in HEK293 cells and stored at $-80{ }^{\circ} \mathrm{C}$. The viral titre was determined by standard plaque assay in HEK293 cells. Confluent mononucleated L6 myoblasts were infected with the adenovirus at $5.5 \mathrm{pfu} / \mathrm{cell}$ for IкB $\alpha \mathrm{S} 32 \mathrm{~A} / \mathrm{S} 36 \mathrm{~A}$ in serum free $\alpha$-MEM for $2 \mathrm{~h}$ at $37{ }^{\circ} \mathrm{C}$. Cells were subsequently maintained in fresh $\alpha$-MEM containing $2 \% \mathrm{FBS}$ at $37{ }^{\circ} \mathrm{C}$ and allowed to differentiate into myotubes prior to experimental use. For some studies, we also used human (LHCN-M2) myotubes. These were cultured in DMEM/M199 medium (4:1) supplemented with penicillin streptomycin $(100 \mu \mathrm{g} / \mathrm{ml})$, FBS $15 \%$ (v/v) HEPES $(20 \mathrm{mM})$, Zinc sulphate $(30 \mathrm{ng} / \mathrm{ml})$, vitamin B12 $(1.4 \mu \mathrm{g} /$ $\mathrm{ml})$, dexamethasone $(55 \mathrm{ng} / \mathrm{ml})$, hepatocyte growth factor, recombinant human $(2.5 \mathrm{ng} / \mathrm{ml})$, and basic FGF $(10 \mathrm{ng} / \mathrm{ml})$ on plastic ware that had been coated overnight with gelatin $(0.1 \%(w / v)$ at room temperature. Confluent myotubes were differentiated by change of culture media to DMEM/medium 199 containing zinc sulphate, HEPES, vitamin B12, insulin $(10 \mu \mathrm{g} / \mathrm{ml})$, and apotransferrin $(100 \mu \mathrm{g} / \mathrm{ml})$ and refreshing this every $48 \mathrm{~h}$ for 9-10 days until myotubes were $70-80 \%$ differentiated.

Prior to use in experiments, L6 myotubes were incubated in serum-containing media that either had or lacked D-glucose $(5 \mathrm{mM})$ as indicated in the figures. For palmitate (PA) treatments, a $100 \mathrm{mM}$ stock solution of the fatty acid was prepared in absolute ethanol as previously reported [28, 29]. This stock was subsequently diluted to a final concentration as indicated in the figure legends by addition to culture media containing $2 \%(\mathrm{w} / \mathrm{v})$ fatty acid-free BSA and allowed to precomplex for $1 \mathrm{~h}$ at $37^{\circ} \mathrm{C}$ before being applied onto myotubes for the periods indicated in the figure legends.

\section{Quantitative real-time PCR, mitochondrial DNA quantification, and analysis of citrate synthase activity}

Myotubes were incubated with glucose, palmitate, and 2-dexoglucose (2DG) or with inhibitors and/or fluorescent dyes as indicated in the figure legends and prepared for RNA extraction, qPCR analysis, and immunoblotting as described previously $[17,25,30,31]$. Briefly, total RNA was extracted from L6 myotubes using the TRizol extraction protocol (Thermo Fisher Scientific, UK). RNA samples were used to prepare cDNA using a qScript cDNA synthesis kit as per manufacturer's instructions and cDNA quantified using the real-time PCR Syber Green based method to establish mRNA abundance. Analysis of mitochondrial DNA (mtDNA) and citrate synthase activity were used as a proxy for mitochondrial mass. For mtDNA quantification, total DNA was extracted from L6 myotubes using a Qiagen DNaesy kit. The mtDNA was quantified by qPCR using primers directed against the mitochondrial ND4 gene and the nuclear-encoded COX4 gene and using the Syber Green method. Data were expressed as a ratio of the $\Delta \Delta C_{\mathrm{t}} \mathrm{ND} 4$ to the $\Delta \Delta C_{\mathrm{t}}$ of COX4. The forward and reverse primer sequences for the different gene targets are detailed in Table 1. Citrate synthase (CS) activity was measured using a kit purchased from Sigma-Aldrich/UK (MAK193). Myotubes were treated as indicated in the figure legend and whole cell extracts prepared at the end of the appropriate treatments. $20 \mu \mathrm{g}$ protein from the cell extract was used for each enzymatic analysis (with measurements being conducted in triplicate for each experimental determination at room temperature). Enzyme activity was measured spectrophotometrically (using an absorbance wavelength of $412 \mathrm{~nm}$ ) using a $\mu$ QUNT BIOTEK plate reader from LabTech UK with readings taken every 3 min over a $60 \mathrm{~min}$ assay period. CS activity was calculated as per manufacturer's instructions.

\section{Subcellular fractionation}

A mitochondrial-enriched membrane fraction was isolated from L6 myotubes using a mitochondria isolation kit (\#89874, Thermo Fisher Scientific) as per manufacturer's instructions. The methodological protocol involves homogenisation of myotubes that have been harvested from $10 \mathrm{~cm}$ tissue culture plates having undergone prior experimental treatments as indicated in the appropriate figure legends in lysis buffer [10 mM HEPES, PH 7.5, $10 \mathrm{mM} \mathrm{KCl,} 0.1 \mathrm{mM}$ EDTA, $0.1 \mathrm{mM}$ DTT, $0.5 \%$ (v/v) Nonidet-P40 and $0.5 \mathrm{mM}$

Table 1 Primer sequences

\begin{tabular}{lll}
\hline GENE & Forward primer & Reverse primer \\
\hline SDHA & GCCACTCACTCTTACACACC & GCACTCCCCATTTTCCATC \\
UCP3 & GTCAAGCAGTTCTACACCCC & TTTCCTCTCGCCTCCAGTTC \\
ANT1 & TCATCTACAGAGCTGCCTAC & TCATCATCCTACGACGGAC \\
IL6 & AGCCACTGCCTTCCCTACTT & GCCATTGCACAACTCTTTTCTC \\
ND4 & GAGGCAACCAAACAGAACGC & ATCATGTTGAGGGTAGGGGGT \\
COX4 & AATGTTGGCTACCAGGGCAC & GGGTAGTCACGCCGATCAAC \\
$\beta-$ ACTIN & TGGAGAAGATTTGGCACCACAC & CAGAGGCATACAGGGACAACAC \\
PGC1 $\alpha$ & TGAACTACGGGATGGCAAC & AAGAGCAAGAAGGCGACAC \\
\hline
\end{tabular}


PMSF and protease inhibitor cocktail]. The homogenised cell material was subject to two differential centrifugation steps and within the final centrifugation step the resulting mitochondrial pellet was washed twice prior to being solubilised in RIPA buffer. The supernatant from the final spin (cytosolic fraction) and the solubilised mitochondrial membrane pellet were stored at $-20{ }^{\circ} \mathrm{C}$ until required.

For isolation of nuclear membranes, L6 myotubes were grown on $10 \mathrm{~cm}$ dishes as described above and, after treatments, washed three times in PBS before being harvested and spun down in a microfuge ( $100 \mathrm{~g}$ for $5 \mathrm{~min})$. The cell pellet was resuspended in lysis buffer and held on ice for 20 min with intermittent mixing prior to being centrifuged at $10,000 \mathrm{~g}$ for $5 \mathrm{~min}$. The resulting supernatant represents a cytosolic fraction. The pelleted nuclei were washed three times in lysis buffer before being resuspended in nuclear extraction buffer (20 mM HEPES PH 7.5, $400 \mathrm{mM} \mathrm{NaCl}$, 1 mM EDTA, 1 mM DTT, 1 mM PMSF with protease inhibitor cocktail) and re-spun at $10,000 \mathrm{~g}$ for $15 \mathrm{~min}$ at $4{ }^{\circ} \mathrm{C}$. The resulting nuclear pellet was resuspended in fresh extraction buffer and stored at $-20{ }^{\circ} \mathrm{C}$ until required.

\section{SDS-PAGE and immunoblotting}

Cell lysates, cytosolic, nuclear, or mitochondrial-enriched fractions (20 $\mu \mathrm{g}$ protein) from L6 myotubes and human LHCN-M2 myotubes were subjected to SDS/PAGE on $10 \%$ resolving gels and transferred onto nitrocellulose membranes (Millipore, Harts, UK), as described previously [27]. Membranes were probed with the following primary antibodies for immunoblot analysis: actin (\#A5060) and tubulin (\#T6074) were obtained from Sigma: ANT-1 (\#ab180715) and PGC1 $\alpha$ (\#ab54481) were from Abcam; IkB $\alpha$ (\#SC371), SDHA (\#SC98253), and GAPDH (\#SC32233) were purchased from Santa Cruz; p65 (\#8242), Akt (\#9272), p-AktSer473 (\#9271S), TOM20 (\# 42406S), HA (\#2367S), COX4 (\#4580S), and GPX1 (\# 3286S) and SOD2 (\#D9V9C) were all purchased from Cell Signalling Technology; DLP1/ Drp1 (\#611112) and OPA1 (\#612607) were from BD Biosciences; and UCP3 (\#GTX112699) from Genetex. Primary antibody detection was performed using appropriate horse-radish peroxidase (HRP) conjugated secondary mouse (\#7076S) or rabbit (\#7074S) antibodies were purchased from Cell Signalling Technology and visualised using enhanced chemiluminescence (Pierce-Perbio Biotech, Tattenhall, UK) on Kodak X-OMAT film (Eastman-Kodak, Rochester, UK). The immunoreactive protein bands were quantified using ImageJ software.

\section{Glucose uptake}

L6 myotubes were incubated with glucose, palmitate and BI605906 for times and at concentrations indicated in the figure legends prior to assaying uptake of $10 \mu \mathrm{M}$ 2-deoxyD- $\left.{ }^{3} \mathrm{H}\right]$-glucose as described previously [27]. Non-specific binding was determined by quantifying cell-associated radioactivity in the presence of $10 \mu \mathrm{M}$ cytochalasin B. Cells were washed and subsequently lysed in $50 \mathrm{mM} \mathrm{NaOH}$ and radioactivity quantified by scintillation counting. Protein concentration in cell lysates was determined using the Bradford reagent [32].

\section{ROS quantification}

For analysis of superoxide, L6 myotubes were subject to experimental treatments as indicated in the figure legends prior to being treated with $5 \mu \mathrm{M}$ Mitosox at $37^{\circ} \mathrm{C}$ in a $5 \%$ $\mathrm{CO}_{2}$ incubator for $30 \mathrm{~min}$. Mitosox is a fluorogenic dye that is specifically targeted to mitochondria in live cells, and whose oxidation by superoxide produces red fluorescence that was quantified using a Clario Star plate reader with absorption/emission maxima: 510/585 nm. In some experiments, L6 myotubes were also treated with Mitotempo (a mitochondrial targeted anti-oxidant) prior to analysis of superoxide.

For determination of hydrogen peroxide $\left(\mathrm{H}_{2} \mathrm{O}_{2}\right)$ under live cell conditions, L6 myotubes were incubated with $5 \mu \mathrm{M}$ MitoPYI (a mitochondrial targeted $\mathrm{H}_{2} \mathrm{O}_{2}$ probe) and $1 \mu \mathrm{M}$ deep red cell tracker at $37{ }^{\circ} \mathrm{C}$ in a $5 \% \mathrm{CO}_{2}$ incubator for $45 \mathrm{~min}$. Myotubes were subsequently imaged using a Zeiss confocal microscope with excitation/emission maxima for MitoPYI set to $488 / 530 \mathrm{~nm}$ and that for the cell tracker at $633 / 647 \mathrm{~nm}$. Captured images were analysed to quantify the fluorescent signal generated by MitoPYI from at least 8-10 different visual fields (40-50 myotubes) per condition per experiment using ImageJ software.

\section{Analysis of cellular respiration and mitochondrial energetics}

For analysis of cellular respiration and mitochondrial energetics in L6 and LHCN-M2 myotubes, we used a Seahorse XF24 analyser. L6 myotubes were cultured on Seahorse culture plates in serum-containing media supplemented with $5 \mathrm{mM}$ D-glucose and/or palmitate at concentrations indicated in the figure legends for $16 \mathrm{~h}$. In some experiments, the culture media were also supplemented with $5 \mathrm{mM}$ 2-deoxyglucose (2-DG), BI605906 (IKK $\beta$ inhibitor) or $2 \mathrm{mM}$ carnitine as indicated prior to analysis of basal respiration, ATPlinked respiration, $\mathrm{H}+$ (proton) leak, maximal respiratory capacity and non-mitochondrial respiration using modulators of cellular respiration (i.e., oligomycin, FCCP (carbonyl cyanide $p$-trifluoromethoxyphenylhydrazone), rotenone, and antimycin as previously described [33]. The various mitochondrial parameters were normalised to protein content/ well within the Seahorse plate. For Seahorse XF analyser 
studies, data points per experimental condition were collected from a minimum of three replicates with each experiment being conducted at least three times.

\section{Mitochondria morphology and live cell mitochondrial imaging}

For analyses of mitochondrial morphology, we stained L6 myotubes with Mitospy Green FM (BioLegend, UK); a green-fluorescent stain that localizes to mitochondria. L6 myotubes were grown on $15 \mathrm{~mm}^{2}$ glass coverslips and following the experimental treatments specified in the figure legends were washed with fresh media and subsequently incubated in medium containing $300 \mathrm{nM}$ Mitospy for $30 \mathrm{~min}$ at $37^{\circ} \mathrm{C}$ in a $95 \% \mathrm{O}_{2} / 5 \% \mathrm{CO}_{2}$ environment. After this incubation period, myotubes were washed with PBS prior to being fixed with $2 \%(w / v)$ paraformaldehyde and mounted in prolonged diamond antifade before being visualised using a Zeiss confocal microscope. Live cell imaging was also used in some of our studies. For these, L6 or LHCN-M2 myotubes were grown and differentiated in eight well chamber slide plates (Ibidia, UK) and having been treated (as indicated in the figure legends) were washed with fresh phenol redfree media prior to incubation with Mitospy. Mitochondrial morphology was then visualised in real time using Zeiss confocal microscope $37^{\circ} \mathrm{C}$ in a $5 \% \mathrm{CO}_{2}$ chamber with excitation/emission set at $480 \mathrm{~nm}$ and $520 \mathrm{~nm}$, respectively. For real-time recording of mitochondrial length, we used the ZEISS ZEN microscope software or Image J. Within each experimental condition, at least 50 myotubes were randomly selected from between 10 and 12 visual fields. Mitochondrial morphology within myotubes was categorised as either spheroid/fragmented in which mitochondria were equal to or less than $1 \mu \mathrm{m}$ in length or tubular/elongated (including being part of a network), where mitochondrial length was greater than $1 \mu \mathrm{m}$. The number of mitochondria in each category within the fields being visualised was then determined and expressed as a percentage.

\section{Analysis of mitophagy}

Mitophagy was quantified using the mitophagy QC approach [34], which involves stable expression of a tandem mCherryGFP tag attached to the outer mitochondrial membrane localization signal of Fis1 (residues 101-152) [34]. The retrovirus harbouring this construct was introduced into L6 myotubes using the approach detailed previously [35]. The L6-GFP-mCherry cells were grown and differentiated on $15 \mathrm{~mm}^{2}$ cover slips and subjected to the treatments detailed in the figure legends prior to being washed and fixed with $3.7 \%$ cell culture grade paraformaldehyde and mounted in prolonged diamond antifade. Cells were visualised using Zeiss 710 confocal microscope. Myotubes expressing the mCherry-GFP construct fluoresce red and green (yellow when confocal images are merged). However, upon increased mitophagy, mitochondria are delivered to lysosomes, where the low pH quenches the GFP signal but not mCherry. Consequently, some of the mitochondria form punctate structures and fluoresce red only and the degree of mitophagy calculated by quantitating their increase using the volocity software.

\section{Statistical analysis}

Statistical analysis was performed using the GraphPad Prism version 7 software using one-way analysis of variance (ANOVA) and Tukey post hoc test for multiple comparisons. Values were considered significant at $P<0.05$.

\section{Results}

\section{Effects of palmitate oversupply on proinflammatory signalling, ROS generation and mitochondrial function in myotubes}

In an attempt to establish the relationship between changes in proinflammatory NFkB signalling, ROS generation, and mitochondrial biology, we initially investigated the effects of modulating palmitate (PA) provision on these parameters. L6 myotubes were incubated in $\alpha$-MEM containing a physiological $(5 \mathrm{mM})$ D-glucose (GLC) concentration in the absence and the presence of increasing concentrations of palmitate (PA; $0.1-0.5 \mathrm{mM}$ ) for $16 \mathrm{~h}$. At the end of this period, the abundance of $\mathrm{IkB} \alpha$ and expression of IL-6 mRNA were monitored as readouts of NFkB signalling. Figure $1 \mathrm{a}, \mathrm{b}$ shows that myotubes exposed to increasing PA concentrations exhibit a dose-dependent reduction in cellular IkB $\alpha$ abundance that was associated with a concomitant increase in IL-6 gene expression. Since the threshold concentration at which PA induced a significant change in $\mathrm{IkB} \alpha$ and IL- 6 gene expression was $0.4 \mathrm{mM}$, all subsequent experiments involving nutrient overloading of myotubes were conducted in the presence of $5 \mathrm{mM}$ GLC and $0.4 \mathrm{mM}$ PA unless otherwise indicated. It is important to stress that combined provision of GLC and PA at these concentrations did not invoke any notable death or loss of terminally differentiated myotubes on culture plates.

A characteristic feature associated with mitochondrial fuel overload is increased cellular ROS [e.g., superoxide $\left(\mathrm{O}_{2}^{-}\right)$and hydrogen peroxide $\left.\left(\mathrm{H}_{2} \mathrm{O}_{2}\right)\right]$, whose generation can promote oxidative damage and metabolic dysfunction. Whilst incubation of myotubes with $5 \mathrm{mM}$ GLC or $0.4 \mathrm{mM}$ PA alone had no significant effect on ROS production in myotubes, the combined presence of both nutrients induced a significant increase in $\mathrm{O}_{2}^{-}$and $\mathrm{H}_{2} \mathrm{O}_{2}$ (Fig. 1c, d). 
A

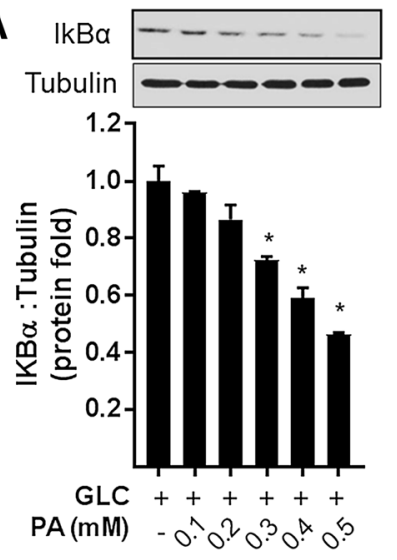

$E$

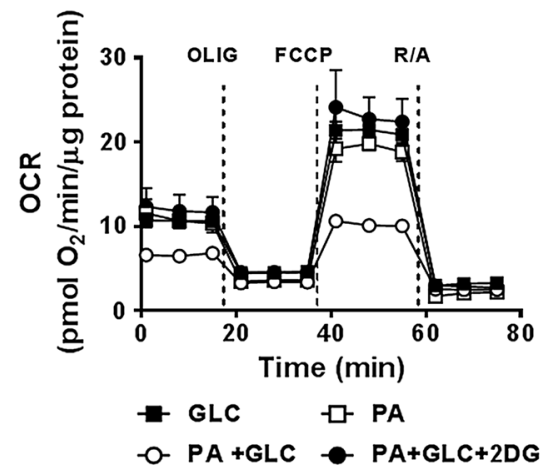

B

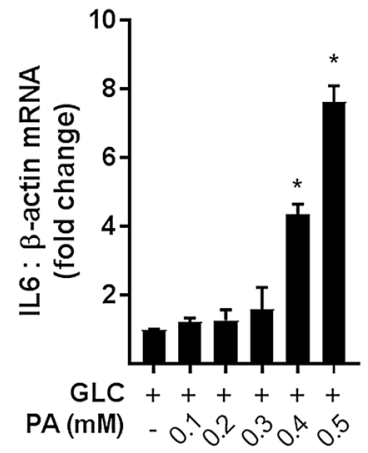

$\mathbf{F}$

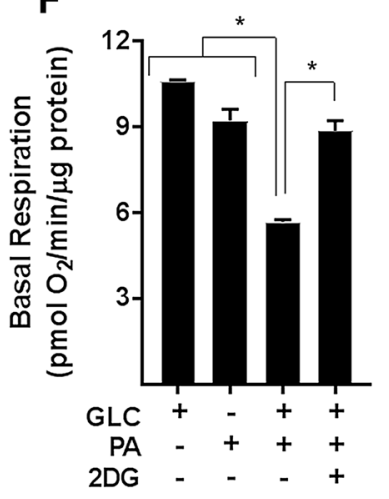

C

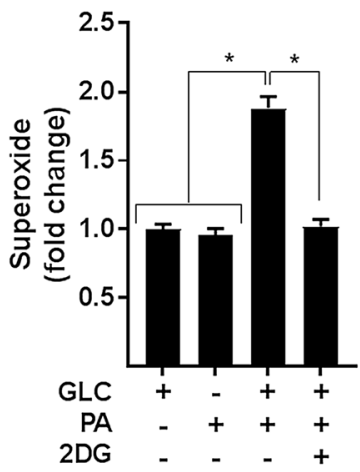

G

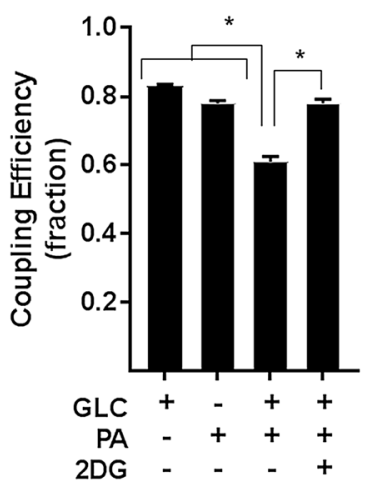

D

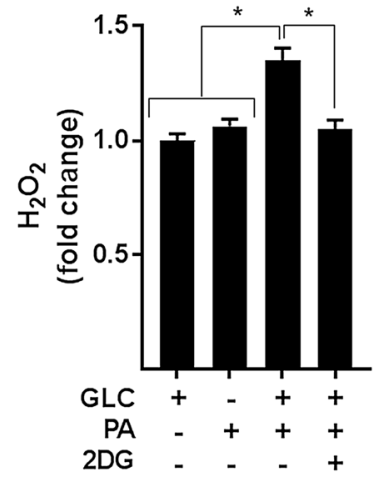

H

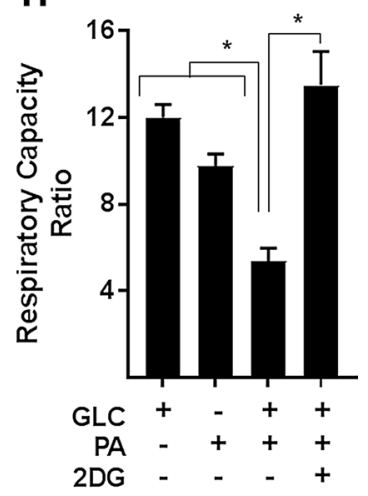

Fig. 1 Cellular fuel overloading induces NFkB inflammatory signalling, ROS (superoxide and $\mathrm{H}_{2} \mathrm{O}_{2}$ ) production and mitochondrial dysfunction. L6 myotubes were incubated with glucose (GLC, $5 \mathrm{mM}$ ) in the absence and presence of palmitate (PA) at doses indicated for $16 \mathrm{~h}$ prior to analysis of (a) cellular IkB $\alpha$ abundance by immunoblotting and $\mathbf{b}$ IL6 and $\beta$-actin mRNA abundance by qPCR. For analysis of superoxide (c) and $\mathrm{H}_{2} \mathrm{O}_{2}$ (d) $\mathrm{L} 6$ myotubes were treated as in (a) with PA $(0.4 \mathrm{mM})$ for $16 \mathrm{~h}$ in absence or presence GLC $(5 \mathrm{mM})$ and/ or 2-deoxyglucose (2DG, $5 \mathrm{mM}$ ) as indicated followed by quantification of ROS using $5 \mu \mathrm{M}$ of either Mitosox or MitoPYI as detailed in methods. For analysis of real-time cellular respiration in L6 myotubes we used a Seahorse XF24 analyser. L6 myotubes were incubated for $16 \mathrm{~h}$ with either GLC $(5 \mathrm{mM})$ or PA $(0.4 \mathrm{mM})$ alone, PA $(0.4 \mathrm{mM}) / \mathrm{GLC}(5 \mathrm{mM})$ together or with a mixture of PA $(0.4 \mathrm{mM}) /$

The notion that this increase in ROS is driven by nutrient overload is strengthened by our demonstration that increases in $\mathrm{O}_{2}^{-}$and $\mathrm{H}_{2} \mathrm{O}_{2}$ are regulated in a PA and GLC concentration-dependent manner (Supplementary Fig. S1 and S2) and that increases in ROS can be restrained in myotubes exposed to 2-deoxyglucose (2DG); a glycolytic inhibitor that severely restricts GLC use as a metabolic fuel (Fig. 1c, d; Supplementary Fig. S1 and S2). Furthermore, we contest that the increase in ROS, we see in response to GLC/PA overloading is principally mitochondrial generated given that treating myotubes with either Mitotempo or MitoQ, two mitochondrial targeted antioxidants, which, respectively, scavenge $\mathrm{O}_{2}^{-}$and quench
GLC $(5 \mathrm{mM}) / 2 \mathrm{DG}(5 \mathrm{mM})$. Oligomycin $(1 \mu \mathrm{M})$, FCCP $(1 \mu \mathrm{M})$ and a rotenone $(1 \mu \mathrm{M}) /$ antimycin-A $(2 \mu \mathrm{M})$ mix were added at times indicated by dotted lines on the Seahorse trace. The trace shown in (e) is a representative readout of oxygen consumption rate (OCR) from a single experiment with measurements (mean $\pm \mathrm{SD}$ ) of triplicate values. Analysis of absolute basal mitochondrial OCR normalised to protein/well (f), coupling efficiency of oxidative phosphorylation (g, determined as the oligomycin sensitivity fraction of the basal respiratory rate) and (h) the respiratory capacity ratio (calculated as a factor of the FCCP-stimulated respiration/oligomycin resistance). The data shown in $(\mathbf{f}-\mathbf{h})$ is the combined analyses of three separate experiments. All data are presented as mean \pm SEM. Asterisks indicate a significant change $(P<0.05)$ to the GLC alone condition or between the indicated bars

$\mathrm{H}_{2} \mathrm{O}_{2}$, effectively suppress increases in both ROS initiated by fuel oversupply (Supplementary Fig. S1D and S2D).

For analysis of real-time mitochondrial respiration in myotubes, we utilised a Seahorse extracellular flux analyser that measures oxygen consumption rates (OCR) before and after addition of compounds that target Complexes I and III of the respiratory chain, the ATP synthase or which function to uncouple mitochondrial oxidative phosphorylation (OXPHOS) to allow analysis of numerous mitochondrial parameters. Figure 1e-h shows that compared to myotubes incubated with either $5 \mathrm{mM}$ GLC or $0.4 \mathrm{mM}$ PA alone, those exposed to both carbon fuels simultaneously had a lower OCR and also exhibited 
a significant reduction in basal respiration, OXPHOS coupling, and respiratory capacity. Strikingly, these respiratory changes are averted in myotubes incubated in media containing GLC and PA, but to which 2DG was also added to inhibit glucose use as a metabolic substrate. To further demonstrate that this amelioration most likely reflects a lowering of mitochondrial substrate load rather than a reduction in substrate competition, we assessed the impact of increasing the PA concentration in the absence of any GLC. Figure 2a shows that whilst the OCR was comparable when myotubes were incubated with either $5 \mathrm{mM}$ GLC or $0.4 \mathrm{mM}$ PA alone and co-provision of GLC and PA at these concentrations induced a significant decline in basal respiration, this reduction could also be recapitulated in myotubes incubated with PA alone, but when presented at a higher concentration $(0.7 \mathrm{mM})$ to increase mitochondrial substrate load. It is also important to stress that incubation of myotubes with PA was conducted in serum-containing media in which carnitine was present at physiological concentrations. Consequently, we believe it unlikely that carnitine would be limiting for mitochondrial uptake and oxidation of PA at the concentrations used in our studies. In line with this view, exogenous supplementation of carnitine $(2 \mathrm{mM})$ to culture media did not enhance or ameliorate the reduction in mitochondrial respiratory capacity that we see in PA/GLC treated myotubes (Fig. 2b).

\section{Effects of mitochondrial fuel overload and glycolytic inhibition on mitochondrial morphology}

The data presented in Fig. 1e-h imply that chronic oversupply of metabolic fuel (GLC + PA) to myotubes impairs mitochondrial respiration and that this can be mitigated when use of GLC as a metabolic fuel was restricted using 2DG as a glycolytic inhibitor. To test whether the reduced respiratory function assayed under fuel-overload conditions was associated with changes in mitochondrial morphology, we subsequently performed live cell imaging to visualise mitochondria using Mitotracker Green. The dye is excluded from nuclei, but accumulates within mitochondria and consequently helps depict the syncytial nature of differentiated L6 myotubes used in our study. Strikingly, in myotubes that had been incubated with GLC or PA alone, the greenfluorescent mitochondrial dye highlights that $\sim 80 \%$ of the mitochondrial population is part of an organised elongated/ tubular network, which becomes structurally fragmented and spheroid in nature when myotubes are subjected to a sustained period of fuel overloading with GLC and PA (Fig. 3a, b). In line with the finding that glycolytic inhibition with 2DG helps preserve mitochondrial respiratory function (Fig. 1e-h), the morphological change in the mitochondrial network caused by substrate overloading was mitigated by 2DG (Fig. 3).
Fig. 2 Effect of glucose/palmitate overloading and carnitine supplementation on mitochondrial function in L6 myotubes. L6 myotubes were incubated with glucose (GLC, $5 \mathrm{mM}$ ), palmitate (PA, $0.4 \mathrm{mM}$ or $0.7 \mathrm{mM})$ or with GLC $(5 \mathrm{mM})$ and PA $(0.4 \mathrm{mM})$ together for $16 \mathrm{~h}$ as shown in (a) or in some experiments (b) when treated with GLC and PA together such treatments were done in the presence of either carnitine (2 mM) or 2-deoxyglucose (2DG, $5 \mathrm{mM}$ ) prior to analysis of real-time cellular respiration using a Seahorse XF24 analyser. All data are presented as mean \pm SEM. Asterisks indicate a significant change $(P<0.05)$ between the indicated bars. NS signifies no significant change
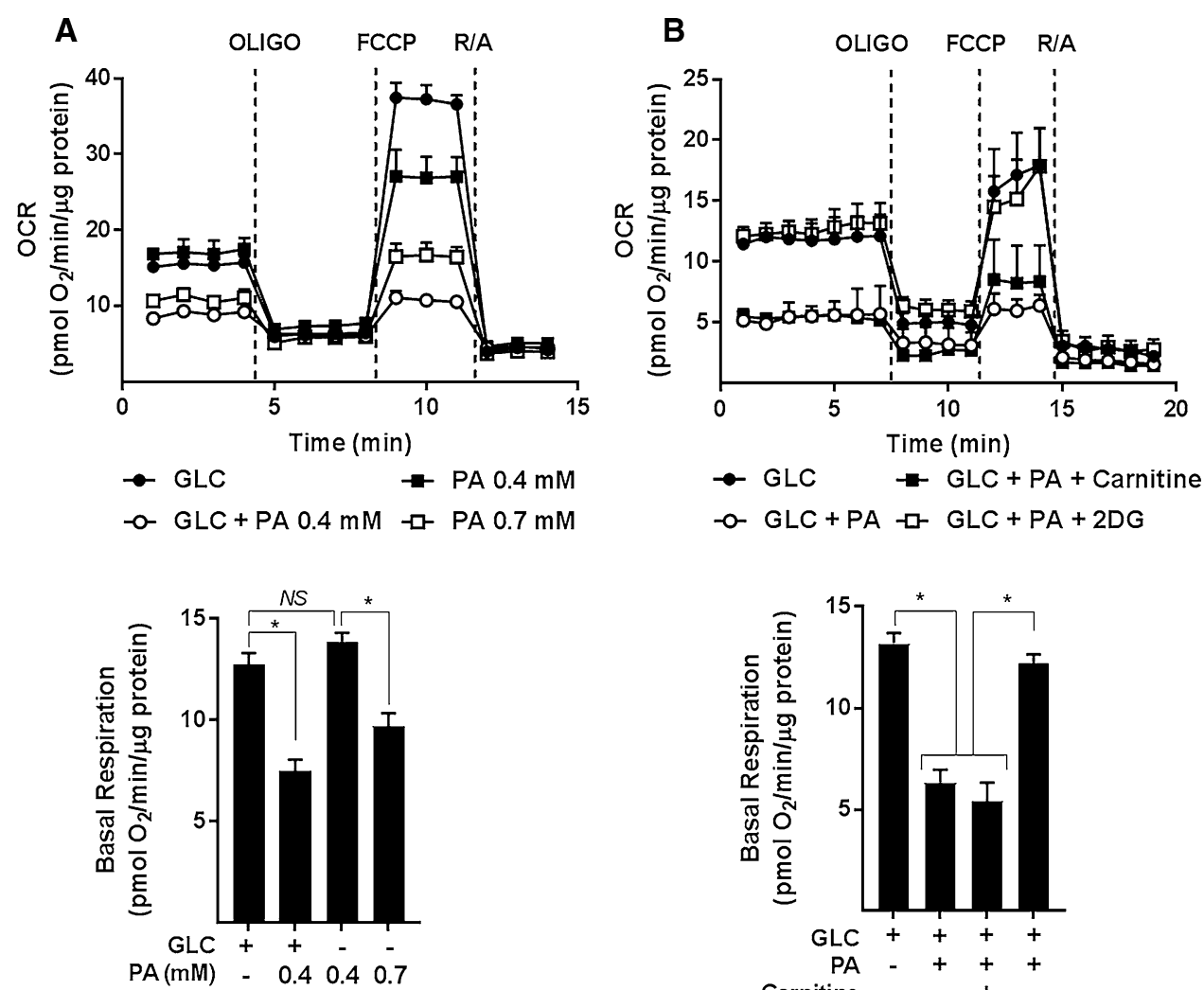

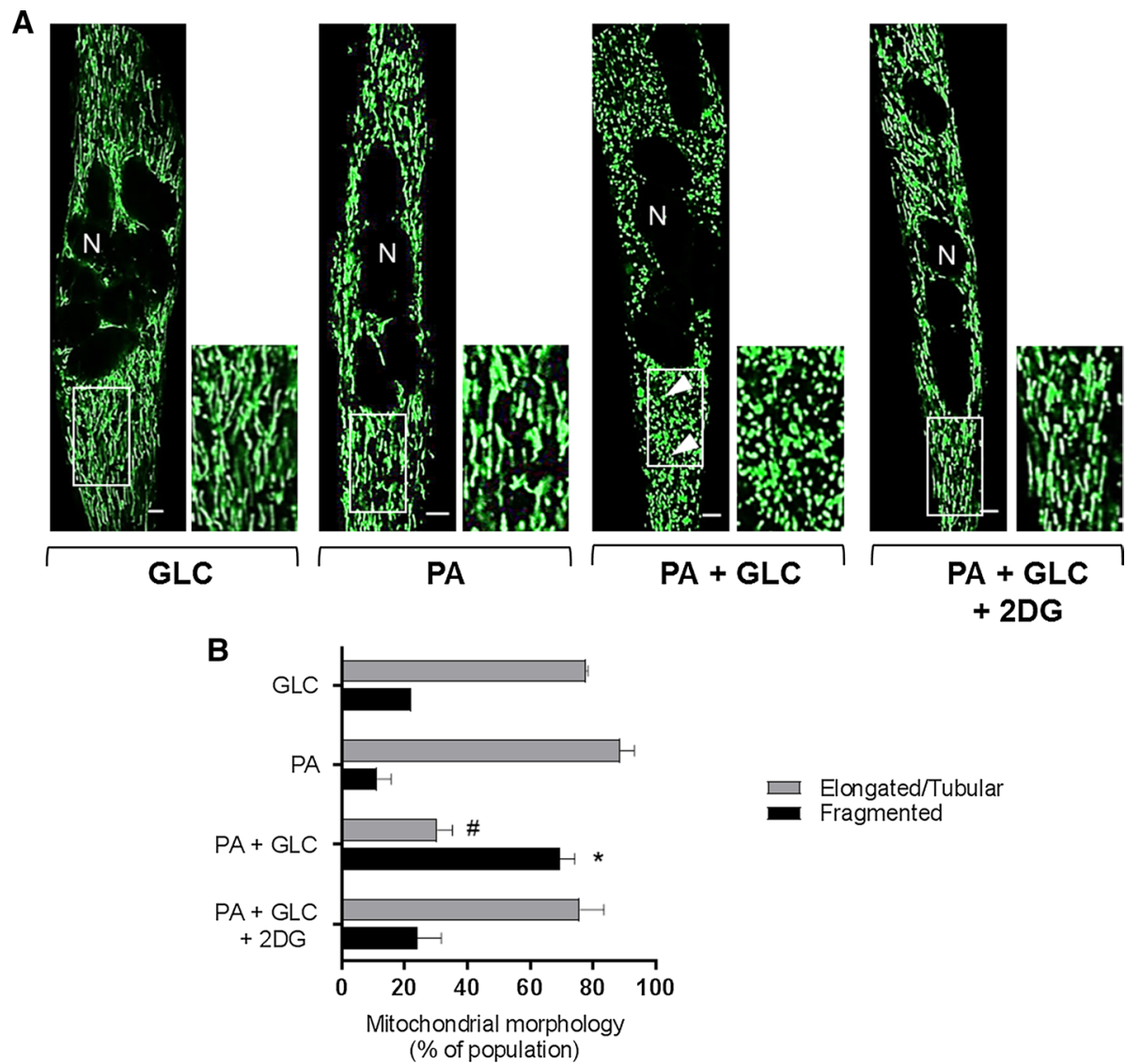

Fig. 3 Effect of glucose/palmitate overloading on mitochondrial morphology in L6 myotubes. L6 myotubes were incubated for $16 \mathrm{~h}$ with either GLC $(5 \mathrm{mM})$ or PA $(0.4 \mathrm{mM})$ alone, PA $(0.4 \mathrm{mM}) /$ GLC $(5 \mathrm{mM})$ together or with a mixture of PA $(0.4 \mathrm{mM}) / \mathrm{GLC}$ $(5 \mathrm{mM}) / 2 \mathrm{DG}(5 \mathrm{mM})$ prior to staining with Mitotracker green (Mitospy) and confocal microscopy. a Confocal images depict mitochondrial morphology in L6 myotubes (the scale bar represents $5 \mu \mathrm{m}$ ). Nuclei $(\mathrm{N})$ are labelled and white boxed areas are magnified to show

\section{Is proinflammatory NFkB signalling a driver of ROS generation in response to nutrient overloading?}

To explore whether the increase in NFkB signalling seen in myotubes subjected to nutrient overload is a contributing factor to mitochondrial ROS production and respiratory dysfunction, we applied two distinct but complimentary strategies. The first utilised BI605906, a potent pharmacological inhibitor that shows high selectivity for $\operatorname{IKK} \beta\left(\mathrm{IC}_{50}\right.$ $=380 \mathrm{nM}$ ) when tested against over 100 kinases in a panel screen [36], whereas the second involved adenoviral mediated expression of a mutant form of $\mathrm{I \kappa B} \alpha(\mathrm{S} 32 \mathrm{~A} / \mathrm{S} 36 \mathrm{~A})$ that functions as a super-repressor of NFkB activity [25]. Mutation of Ser32 and Ser36 to alanine renders IкB $\alpha$ resistant to IKK phosphorylation, thereby protecting it from proteasomal degradation and retaining its capacity to hold $\mathrm{NF \kappa B}$ in an inhibited state. To test the effectiveness of each strategy, we initially assayed the ability of BI605906 and the IאB $\alpha^{\mathrm{S32A} /}$ differences in morphology. The arrow heads depict fragmented mitochondria. b Mitochondrial length was quantified using the imaging software and presented as elongated/tubular if greater than $1 \mu \mathrm{m}$ and fragmented if $<1 \mu \mathrm{m}$ in length. Data are presented as mean \pm SEM from a minimum of three separate experiments. Asterisks indicate a significant change $(P<0.05)$ between the black filled bars, whereas the hash $(\#)$ indicates a significant difference $(P<0.05)$ between the grey filled bars

${ }^{\mathrm{S} 36 \mathrm{~A}}$ mutant to suppress NFkB activation in response to nutrient overload in L6 myotubes. The data in Fig. 4a, b show that the reduction in $\mathrm{IkB} \alpha$ instigated by a $16 \mathrm{~h}$ period of nutrient (GLC + PA) overloading in myotubes was halted by BI605906 or by cellular expression of the IkB $\alpha$ mutant. Moreover, consistent with this observation, both BI605906 and $\mathrm{I \kappa B} \alpha^{\mathrm{S} 32 \mathrm{~A} / \mathrm{S} 36 \mathrm{~A}}$ expression attenuated nuclear localisation of the p65 subunit of NFkB and reduced transcription of the IL-6 gene (an NFkB target gene) that is otherwise seen in GLC+PA-overloaded myotubes (Fig. 4c, d).

Analysis of ROS production in L6 myotubes revealed that in the absence of PA provision, BI605906 per se has no detectable effect upon cellular $\mathrm{O}_{2}^{-}$or $\mathrm{H}_{2} \mathrm{O}_{2}$, but significantly reduces the increase in both species when myotubes were subjected to chronic nutrient oversupply (Fig. 5a, b). Similarly, repressing NFkB activation by expression of $\mathrm{I} \kappa \mathrm{B} \alpha^{\mathrm{S} 32 \mathrm{~A} / \mathrm{S} 36 \mathrm{~A}}$ blocked the increase in ROS triggered by nutrient overload (Fig. 5c, d). It is noteworthy that this 


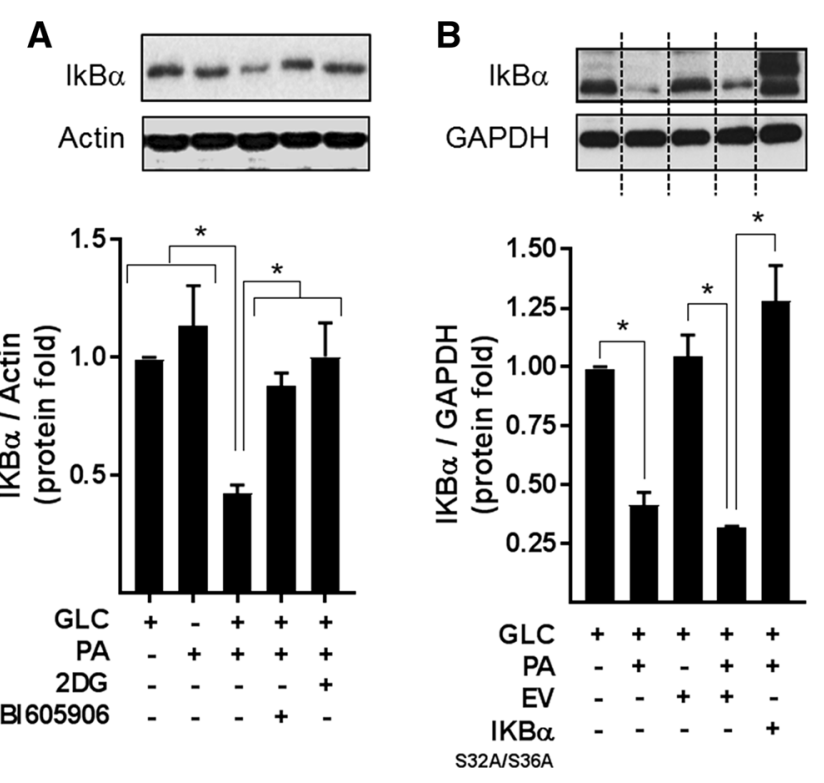

Fig. 4 Effects of suppressing NFkB on inflammatory signalling induced by nutrient overload in L6 myotubes. a L6 myotubes were incubated for $16 \mathrm{~h}$ with either GLC $(5 \mathrm{mM})$ or PA $(0.4 \mathrm{mM})$ alone, PA $(0.4 \mathrm{mM}) / \mathrm{GLC}(5 \mathrm{mM})$ together or with a mixture of PA $(0.4 \mathrm{mM}) / \mathrm{GLC}(5 \mathrm{mM}) / 2 \mathrm{DG}(5 \mathrm{mM})$ in the absence or presence of BI605906 $(10 \mu \mathrm{M})$, an IKK $\beta$ inhibitor prior to analysis of cellular $\mathrm{IkB} \alpha$ abundance by immunoblotting. b L6 myoblasts were infected with an adenoviral vector expressing HA-tagged non-phosphorylatable $\mathrm{IkB} \alpha(\mathrm{S} 32 \mathrm{~A} / \mathrm{S} 36 \mathrm{~A})$ or one expressing an empty vector (EV). Cells

nutrient-induced increase in ROS is accompanied by an attendant increase in the expression of anti-oxidant enzymes [superoxide dismutase 2 (SOD2), catalase and glutathione peroxidase (GPX1)], which most likely forms part of a cellular defence mechanism designed to help limit oxidative damage/stress under these circumstances (Supplementary Fig. S2E). Intriguingly, however, whilst the elevated ROS generation induced by cellular over-nutrition was restrained by BI605906 (Fig. 5a, b), the inhibitor did not to suppress the increased expression of SOD2, catalase or GPX1 (Supplementary Fig. S2E), suggesting that expression of these enzymes was likely to be regulated by mechanisms that are distinct to those involved in promoting generation of $\mathrm{O}_{2}{ }^{-}$and $\mathrm{H}_{2} \mathrm{O}_{2}$.

\section{Is increased NFkB signalling a causal factor for mitochondrial dysfunction in myotubes during nutrient overload?}

Impaired mitochondrial bioenergetics can result in the excessive generation of ROS during the process of OXPHOS. Since inhibition of NFkB signalling suppresses the increase in cellular ROS associated with nutrient overload, we subsequently tested whether this might be linked to improved mitochondrial function. The data in Fig. 6 show the effects of

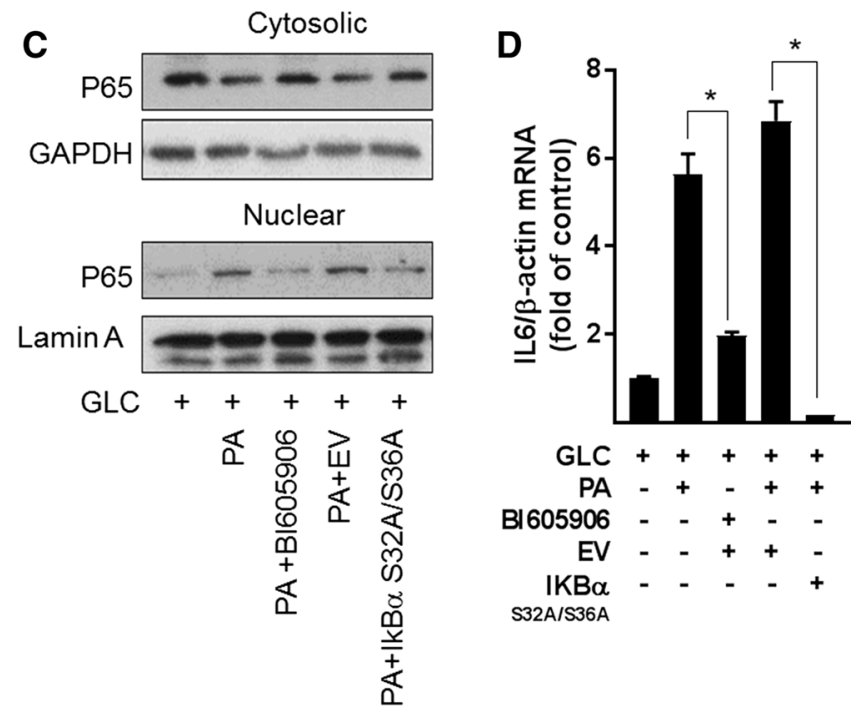

were allowed to differentiate prior to treatment of cells with GLC, PA and 2DG and analysis of $\operatorname{IkB} \alpha$ abundance as described in (a). L6 myotubes were treated with GLC $(5 \mathrm{mM})$, PA $(0.4 \mathrm{mM})$, BI605906 $(10 \mu \mathrm{M})$ and adenoviral vectors as indicated prior to (c) subcellular fractionation and analyses of cytosolic and nuclear NFkB p65 abundance by immunoblotting or $\mathbf{d}$ analysis of IL- 6 gene expression. All data are presented as mean \pm SEM from a minimum of three separate experiments. Asterisks indicate a significant change $(P<0.05)$ between the indicated bars

inhibiting NFkB signalling in L6 myotubes (using BI605906 and by cellular expression of the I $\mathrm{KB} \alpha^{\mathrm{S} 32 \mathrm{~A} / \mathrm{S} 36 \mathrm{~A}}$ super-repressor) upon mitochondrial function. In line with the findings presented in Fig. 1e-h, sustained (16 h) exposure of myotubes to nutrient excess (GLC and PA) induced a significant decline in the basal and maximal respiratory rate, which was associated with a reduction in ATP-linked respiration and a modest decline in mitochondrial proton leak (Fig. 6a-j). Strikingly, these nutrient-induced disturbances in mitochondrial respiration were ameliorated if activation of the IKK $\beta$-NFkB signalling axis by nutrient excess was repressed by BI605906 in a dose-dependent manner (Fig. 6a-e and Supplementary Fig. S3) or expression of the IкB $\alpha^{\mathrm{S} 32 \mathrm{~A} / \mathrm{S} 36 \mathrm{~A}}$ mutant (Fig. 6f-j). Notably, the improved respiratory drive that we see under these circumstances is associated with a significant increase in mitochondrial proton leak (Fig. 6e, j) potentially signifying increased mitochondrial uncoupling that would also help offset ROS generation.

\section{Effects of repressing NFkB signalling on mitochondrial morphology and expression of proteins important for mitochondrial function}

To assess whether the improved respiratory capacity that was seen upon inhibiting NFkB signalling in nutrient-overloaded 


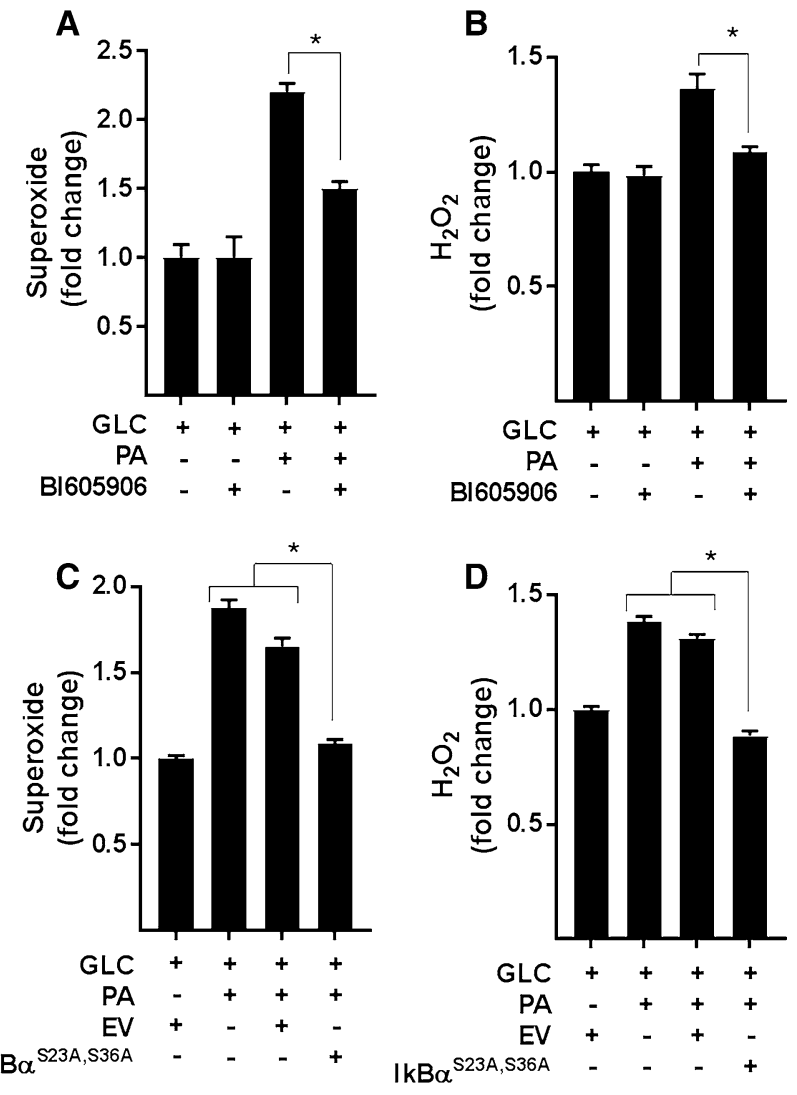

Fig. 5 Effects of NFkB antagonism on ROS production. a, b L6 myotubes were incubated for $16 \mathrm{~h}$ with either GLC $(5 \mathrm{mM})$ or PA $(0.4 \mathrm{mM})$ alone, PA $(0.4 \mathrm{mM}) / \mathrm{GLC}(5 \mathrm{mM})$ together in the absence or presence of BI605906 $(10 \mu \mathrm{M})$ or subject to these treatments having been infected with an adenoviral vector expressing HA-tagged non-phosphorylatable IkB $\alpha(\mathrm{S} 32 \mathrm{~A} / \mathrm{S} 36 \mathrm{~A})$ or one expressing an empty vector $(\mathrm{EV}) \mathbf{c}, \mathbf{d}$ prior to analysis of superoxide and hydrogen peroxide. All data are presented as mean \pm SEM from four separate experiments. Asterisks indicate a significant change $(P<0.05)$ between the indicated bars

myotubes was associated with changes in mitochondrial morphology or mitochondrial mass we subsequently performed live cell imaging of mitochondria stained with Mitotracker Green and also assessed cellular mitochondrial DNA content. Figure $7 \mathrm{a}$ shows that repressing NFkB signalling with BI605906 helps attenuate the fragmentation of the elongated/ tubular mitochondrial network that is otherwise seen when myotubes are chronically exposed to nutrient excess. qPCR analysis was used to determine mitochondrial DNA copy number by quantifying the relative abundance of the mitochondrial encoded NADH dehydrogenase 4 (ND4) gene to that of COX4 (a nuclear-encoded gene). Figure 7b, c shows that irrespective of whether myotubes were subject to nutrient overload or not, or whether they were treated with 2DG or BI605906, we observed no significant differences in mitochondrial DNA or citrate synthase activity (a representative nuclear-encoded mitochondrial enzyme) in response to the various experimental manipulations. Both these measures are considered a proxy of mitochondrial mass.

Whilst mitochondrial mass in L6 myotubes was unaltered following a $16 \mathrm{~h}$ period of nutrient overload, the reduced respiratory rate seen under these circumstances was associated with a significant reduction in the abundance of proteins with key roles in mitochondrial bioenergetics. These include uncoupling protein 3 (UCP3), mitochondrial ADP-ATP translocase (ANT1) and succinate dehydrogenase (SDHA) as well as PGC1 $\alpha$, which has a major role in regulating mitochondrial biogenesis and function (Fig. 7d). Our analysis reveals that the decline in these proteins may have been driven by a decrease in gene expression based on the reduced mRNA abundance that, respectively, encode the four proteins (Fig. 7e). Whilst we saw no change in the protein abundance of Cox 4.1 (a subunit of Cytochrome $\mathrm{C}$-oxidase that functions within the mitochondrial respiratory chain) in myotubes exposed to both GLC and $0.4 \mathrm{mM} \mathrm{PA}$, we have shown previously that its cellular abundance declines dramatically when the presence of PA in GLC-containing media is raised above $0.4 \mathrm{mM} \mathrm{[17,}$ 31]. This latter finding may potentially signify that the expression and stability of Cox 4.1 may have a slightly higher tolerance for nutrient stress than some of the other mitochondrial proteins that we have investigated. Significantly, however, the loss seen in UCP3, ANT1, SDHA, and PGC1 $\alpha$ protein in fueloverloaded myotubes was ameliorated by not only pharmacological repression of the IKK $\beta$-NFkB signalling axis with BI605906, but also by restraining GLC metabolism using 2DG (Fig. 7d, e).

It is plausible that the increased respiratory drive seen in myotubes that have been subject to fuel overloading, but in which activation of the NFkB pathway has been blunted (Fig. 6) is a benefit derived from suppressing mitochondrial ROS production (Fig. 5). However, our analysis indicates that whilst exposing myotubes to two distinct mitochondrial targeted anti-oxidants (Mitotempo and Mito Q) limits fragmentation of the mitochondrial network induced by nutrient oversupply, neither compound could rescue the loss in UCP3, ANT1, or PGC1 $\alpha$ expression or prevent the decline in mitochondrial respiratory capacity (Supplementary Fig. S4). However, we did see some recovery in the expression of SDHA. We are mindful that ROS production can also occur at extra-mitochondrial sites such as via NADPH oxidase (NOXII) in the cytosol, but targeting NOXII with a cell permeable inhibitor, VAS2870, also proved ineffective in countering the decline in respiratory capacity caused by nutrient excess (Supplementary Fig. S4).

\section{Effects of mitochondrial substrate overload on mitophagy}

Since chronic oversupply of metabolic fuel promotes mitochondrial dysfunction, we postulated that under such 

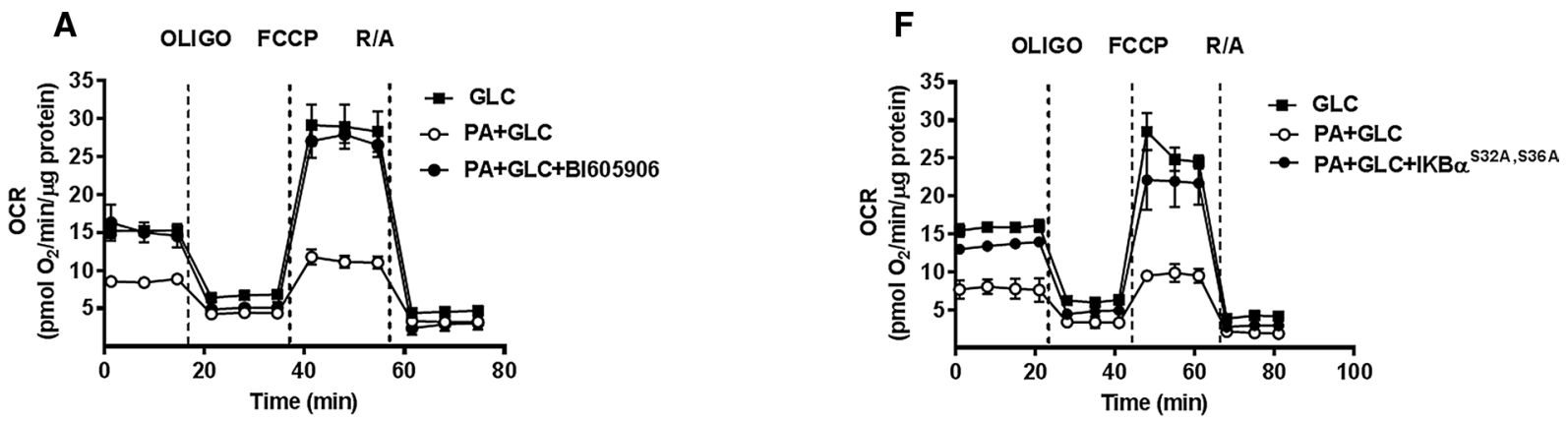
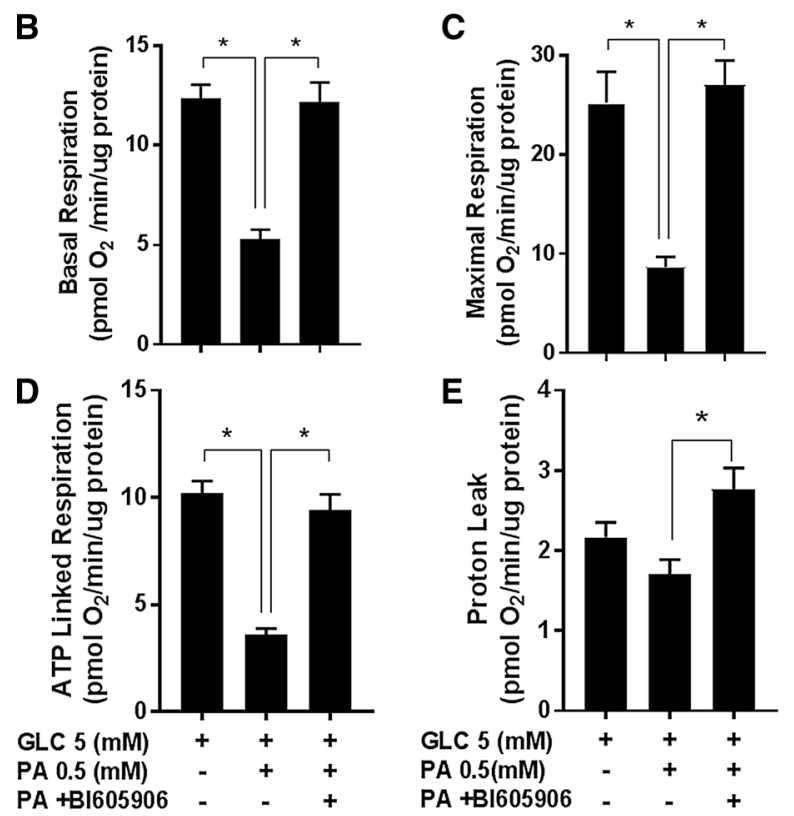

Fig. 6 Effect of suppressing NFkB activation in response to cellular fuel overloading on mitochondrial respiration. a-e L6 myotubes were incubated for $16 \mathrm{~h}$ with either GLC $(5 \mathrm{mM})$ or PA $(0.4 \mathrm{mM})$ alone, PA $(0.4 \mathrm{mM}) / \mathrm{GLC}(5 \mathrm{mM})$ together in the absence or presence of BI605906 $(10 \mu \mathrm{M})$ or $\mathbf{f}-\mathbf{j}$ subject to these treatments having been infected with an adenoviral vector expressing HA-tagged non-phosphorylatable $\mathrm{IkB} \alpha(\mathrm{S} 32 \mathrm{~A} / \mathrm{S} 36 \mathrm{~A})$ or one expressing an empty vector (EV) prior to analysis of real-time cellular respiration in L6 myotubes using a Seahorse XF24 analyser. Oligomycin $(1 \mu \mathrm{M})$, FCCP $(1 \mu \mathrm{M})$ and a rotenone $(1 \mu \mathrm{M})$ /antimycin- $\mathrm{A}(2 \mu \mathrm{M})$ mix were added at times

circumstances, there might be an increase in mitophagy to help clear damaged/dysfunctional mitochondria. To test this hypothesis, we initially monitored expression of proteins implicated in the control of mitochondrial dynamics in a mitochondrial-enriched membrane fraction isolated from myotubes that had been subject to fuel overloading in the absence and presence of BI605906. Figure 8a shows that sustained exposure of myotubes to $5 \mathrm{mM} \mathrm{GLC}$ and $0.4 \mathrm{mM}$ PA induced a very modest increase in the mitochondrial abundance of Drp1, a GTPase that facilitates mitochondrial fission. This increase in mitochondrialassociated Drp1 may have resulted from its recruitment from a cytoplasmic pool, which showed a corresponding
G
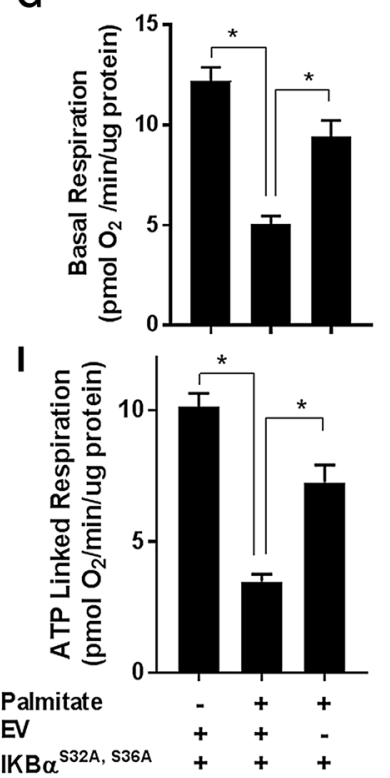

H

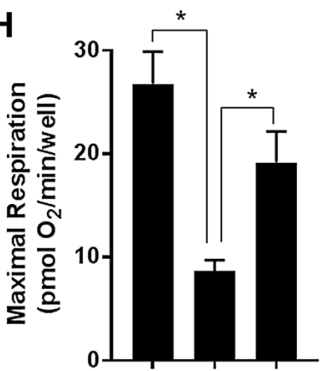

$\mathbf{J}$

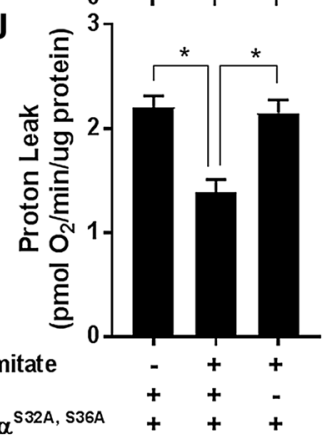

indicated by dotted lines. a, f show representative readouts of oxygen consumption rate (OCR) from a single experiment with measurements (mean $\pm \mathrm{SD}$ ) of triplicate values. b, $\mathbf{g}$ Depict basal mitochondrial oxygen consumption rate (OCR), $\mathbf{c}, \mathbf{h}$ maximal respiration (OCR after FCCP stimulation), d, i ATP-linked respiration (oligomycin-sensitive OCR) and $\mathbf{e}-\mathbf{j}$ proton leak (oligomycin resistance rate). Data are presented as mean \pm SEM from five independent experiments. Asterisks indicate a significant change $(P<0.05)$ between the indicated bars

decline in its abundance. By contrast, we noted a loss in mitochondrial mitofusin 2 (MFN2), a GTPase resident on the outer mitochondrial membrane involved in mitochondrial clustering and fusion (Fig. 8a). The relative changes in mitochondrial Drp1 and MFN2 is consistent with a shift in mitochondrial dynamics that favours increased fission and would be in line with our morphological analysis (Fig. 7a). In contrast, the observed changes in mitochondrial-associated Drp1 and MFN2 abundance were repressed in fuel-loaded myotubes that had been treated with BI605906 and would fit with the reduced mitochondrial fragmentation that we see (Fig. 7a). No notable differences were observed for mitofusin 1 (MFN1) or optic 
A
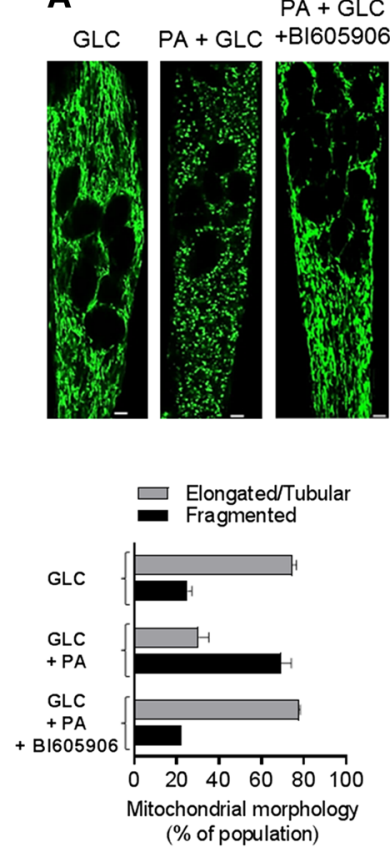

B
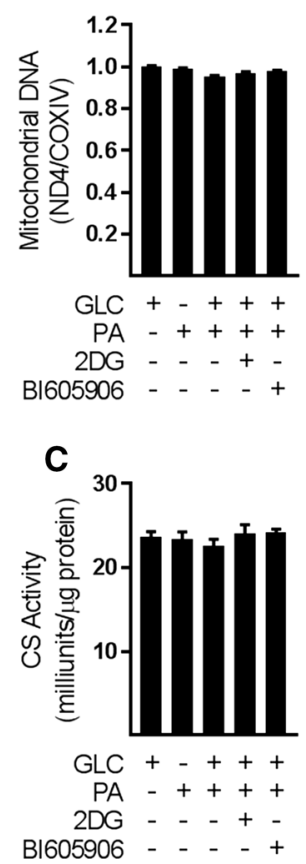

D

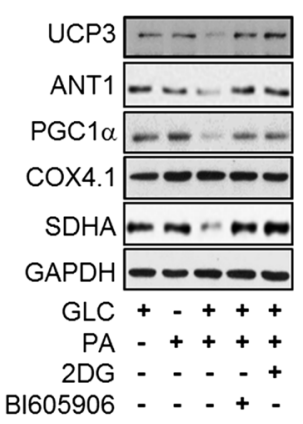

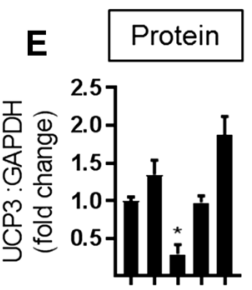
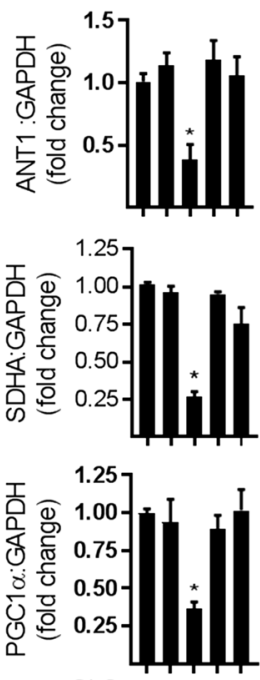

$\mathrm{GLC}+-+++$

$\mathrm{PA}-++++$

$2 \mathrm{DG}$ - . - +

Bl605906 _ - . +
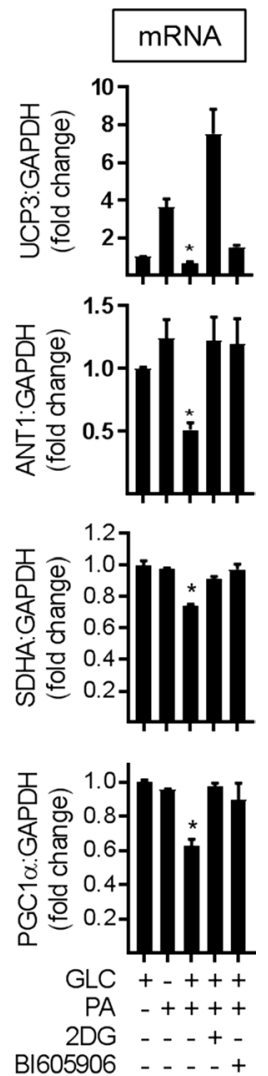

Fig. 7 Effect of suppressing NFkB activation in response to cellular fuel overloading on mitochondrial morphology, mitochondrial proteins and gene expression. L6 myotubes were incubated with GLC $(5 \mathrm{mM}), \mathrm{PA}(0.4 \mathrm{mM}), 2 \mathrm{DG}(5 \mathrm{mM})$ and BI605906 $(10 \mu \mathrm{M})$ for $16 \mathrm{~h}$ in the combinations indicated in the various experimental data panels prior to a analysis and quantification of mitochondrial morphology using Mitotracker green (Mitospy) by confocal microscopy (the scale bar represents $5 \mu \mathrm{m}$ ), b mitochondrial DNA copy number by qPCR, c citrate synthase (CS) activity and (d, e), analysis of mitochondria protein and mRNA abundance (UCP3, ANT1, PGC1 $\alpha$, SDHA, and COX4.1) which was normalised to GAPDH. All graphical bar data are presented as mean \pm SEM from four separate experiments. Asterisks indicate a significant change $(P<0.05)$ to the GLC alone condition atrophy-1 protein (OPA-1) in the mitochondrial and cytosolic fractions that we examined (Fig. 8a).

To assess whether increased fragmentation/fission in response to nutrient excess also increases mitophagy, we utilised myotubes stably expressing a tandem mCherryGFP tag attached to the outer mitochondrial membrane localization signal of Fis1 (residues 101-152) [34]. Figure $8 \mathrm{~b}$, c shows representative field images that highlight (red) the presence of mitophagic objects in myotubes incubated with GLC or PA alone, which most likely reflects basal mitophagy (see arrow heads within the magnified regions from the box inserts). Whilst this approach does not quantitatively assess mitophagic flux, it indicates that the combined provision of GLC and PA induces a significant increase in mitophagic particles (Fig. 8d, f) whose appearance is restrained upon cotreatment of myotubes with BI605906 (Fig. 8e, f).

\section{Effects of suppressing NFkB signalling in substrate-loaded myotubes on insulin sensitivity}

A number of previous studies have shown that sustained exposure of muscle cells to nutrient (glucose and fatty acid) excess impairs insulin action and have linked this to disturbances in mitochondrial function. To assess whether preserving the respiratory capacity and integrity of the mitochondrial network in nutrient overloaded myotubes by inhibition of NFkB signalling improves insulin action, we assessed insulin-stimulated Akt phosphorylation and glucose uptake as readouts. Figure 9a shows that insulin induces a robust increase in $\mathrm{Akt}^{\mathrm{Ser} 473}$ phosphorylation/activation that is blunted significantly (by 58\%) in myotubes that have been subject to GLC/PA oversupply (Fig. 9b). Associated with this loss in Akt-directed insulin, signalling was a substantial loss in insulin-stimulated glucose uptake (Fig. 9c). However, 


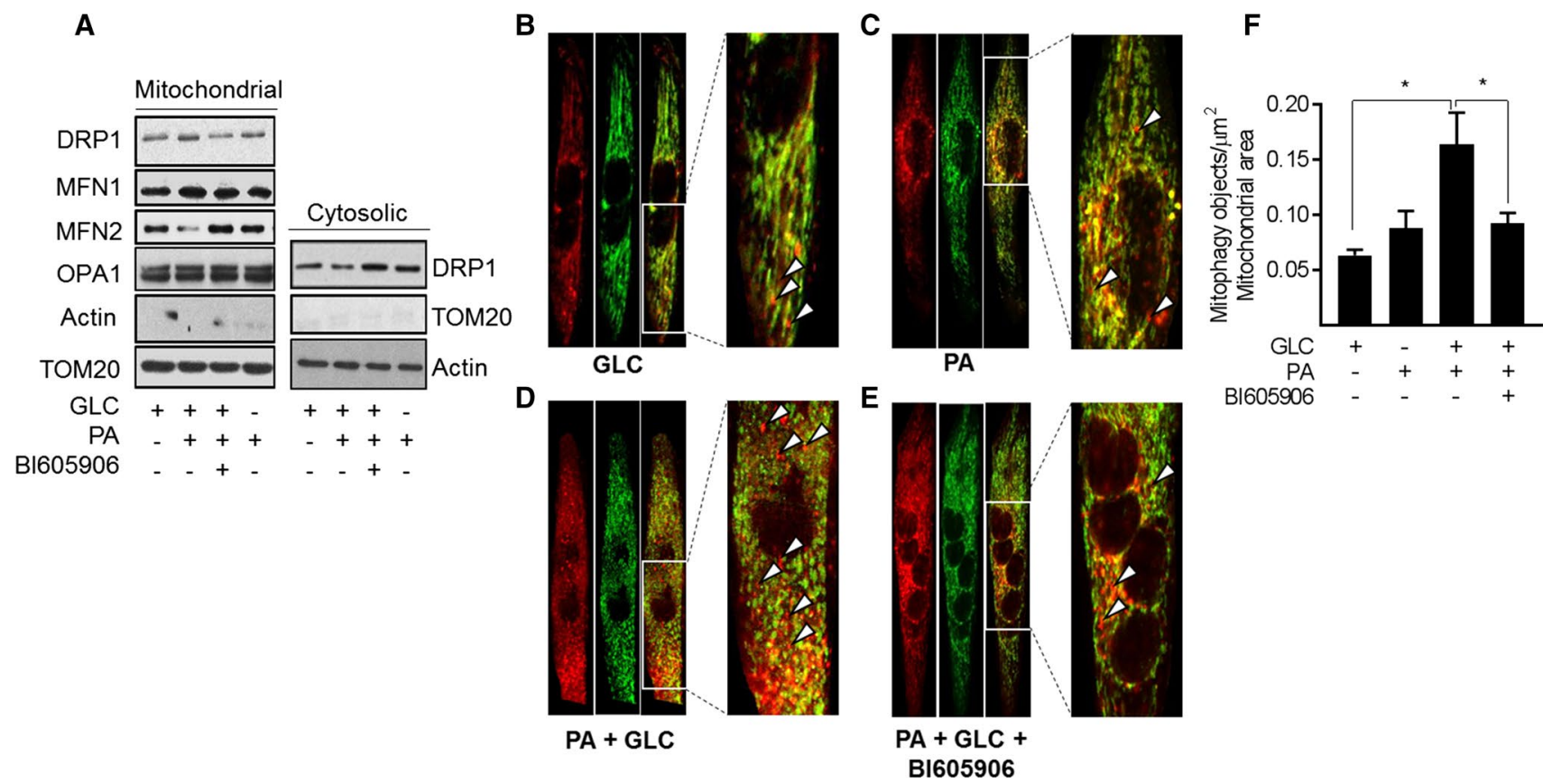

Fig. 8 Effects of cellular fuel overloading on mitophagy and upon proteins linked to mitochondrial dynamics. Wild type L6 myotubes or those stably expressing a retroviral vector encoding a GFP-mCherryFis1 mitophagy reporter were incubated with GLC (5 mM), PA $(0.4 \mathrm{mM})$ and BI605906 $(10 \mu \mathrm{M})$ for $16 \mathrm{~h}$ as indicated prior to: a subcellular fractionation and isolation of a cytosolic and mitochondrialenriched membrane fraction for immunoblotting with antibodies to

cotreatment of fuel-loaded myotubes with BI605906 or

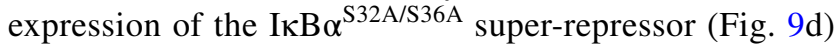
not only mitigates the reduction in insulin-stimulated Akt phosphorylation (Fig. 9a, b, d, e), but also partially rescues the loss in hormone-stimulated glucose uptake (Fig. 9c, f).

\section{Discussion}

We have previously shown that heightened NFkB signalling associated with sustained nutrient oversupply, as occurs during obesity, plays an important role in lipid-induced insulin resistance and metabolic dysfunction in skeletal muscle both in vitro and in vivo [17]. Precisely how an increase in NFkB signalling mechanistically links to changes in muscle insulin action is poorly understood, but we postulate that disturbances in mitochondrial homeostasis, driven by an increase in proinflammatory signalling, may potentially represent an important component within this link. This proposition is based on the view that whilst mitochondria play a crucial role in balancing energy supply with demand under normal circumstances, they become functionally compromised when supply of metabolic fuel chronically exceeds cellular energy demand. Our results indicate that the sustained oversupply of both GLC and PA imposes a major substrate proteins shown. b-e Fixing and confocal imaging to visualise and $\mathbf{f}$ quantifying (using the Volocity software) mitophagy in L6 myotubes. Data (mean \pm SEM) in (f) are from five separate experiments. Asterisks indicate a significant change $(P<0.05)$ between the indicated bars. Boxed sections in panels $\mathbf{b}, \mathbf{c}, \mathbf{d}$ and $\mathbf{e}$ have been expanded to highlight mitophagic particles, some of which are depicted by the white arrow heads

burden on mitochondria in L6 myotubes and that this promotes (1) a reduction in mitochondrial respiratory capacity, (2) reduced expression of key mitochondrial proteins, (3) increased generation of ROS and (4) increased mitochondrial fragmentation and mitophagy. It is also important to highlight that these observations are not just restricted to L6 myotubes, but can also be demonstrated in LHCN-M2 myotubes; a myogenic cell line obtained from skeletal muscle of a healthy male human subject (Supplementary Fig. S5). These nutrient-induced changes collectively signal a marked loss in mitochondrial integrity/function that impact negatively upon myocellular energy metabolism and, by extension, upon processes involved in fuel utilisation, such as glucose uptake and its regulation by insulin. Strikingly, our data indicate that pharmacological or genetic inhibition of the IKK-NFkB axis not only obviates detrimental changes in mitochondrial morphology and function, but also helps ameliorate disturbances in insulin signalling and insulin-dependent glucose uptake. Furthermore, allied to these observations, it is worth stressing that withholding either GLC or PA from the media or inhibiting GLC use as a metabolic fuel in the presence of PA not only averts activation of the NFkB pathway but antagonises mitochondrial fragmentation and the loss in respiratory function (Figs. 1b, 3a, 4a). These findings imply that whilst myotubes can efficiently 
A

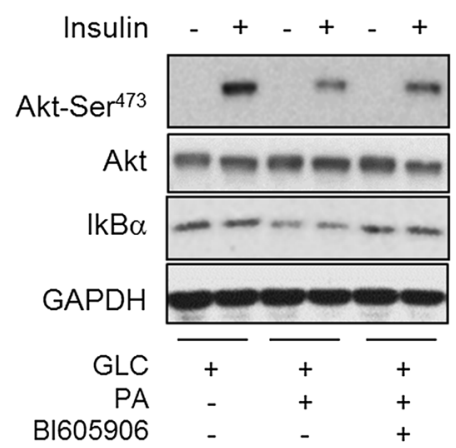

B

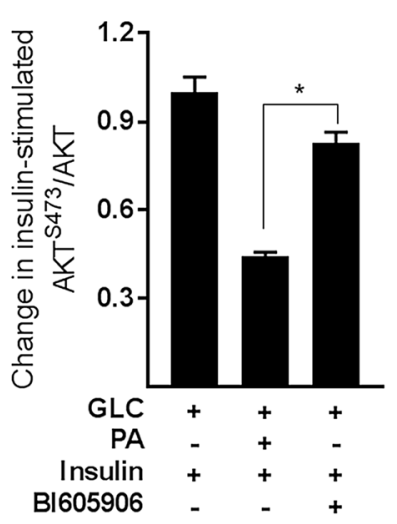

E

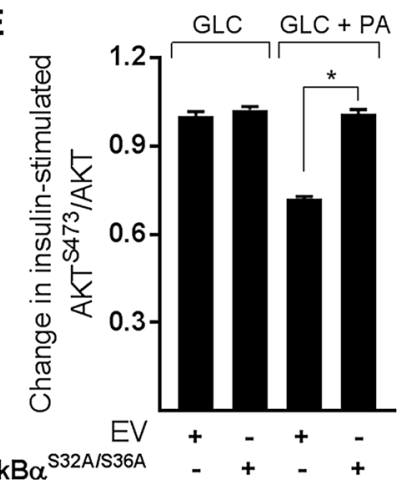

C

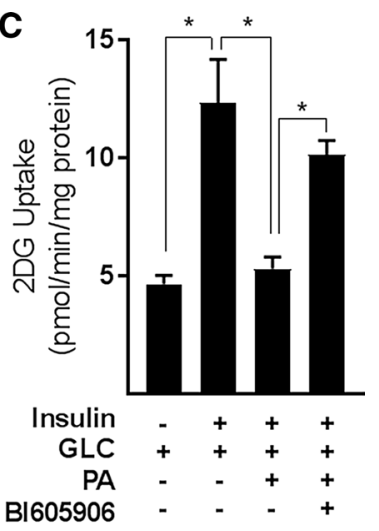

$\mathbf{F}$

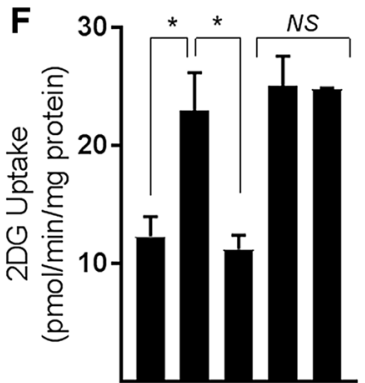

$\mathrm{GLC}+++++$

Insulin -++++

PA - -+-+

$\mathrm{EV}+++-$

$k B \alpha^{\text {S32A/S36A }}-\quad-++$
Fig. 9 Effects of suppressing NFkB signalling in response to cellular fuel overloading on insulin-stimulated PKB phosphorylation and glucose uptake in L6 myotubes a-c L6 myotubes were incubated for $16 \mathrm{~h}$ with GLC $(5 \mathrm{mM})$ or PA $(0.4 \mathrm{mM})$ alone or with PA $(0.4 \mathrm{mM}) / \mathrm{GLC}$ $(5 \mathrm{mM})$ together in the absence or presence of BI605906 $(10 \mu \mathrm{M})$. Alternatively, $\mathbf{d}-\mathbf{f}$ muscle cells were subject to incubation with GLC and PA as indicated having been infected with an adenoviral vector expressing HA-tagged non-phosphorylatable $\mathrm{IkB} \alpha(\mathrm{S} 32 \mathrm{~A} / \mathrm{S} 36 \mathrm{~A})$ or one expressing an empty vector (EV) prior to acute stimulation with insulin $(20 \mathrm{nM})$ for $15 \mathrm{~min}$ (Akt signalling experiments) or insulin $(100 \mathrm{nM})$ for $20 \mathrm{~min}$ for glucose uptake studies. Following insulin stimulation, cells were either lysed for immunoblotting using antibodies to proteins indicated $(\mathbf{a}, \mathbf{d})$ or used for assay of glucose uptake. The data are mean \pm SEM. from 5 separate experiments. Asterisks indicate a significant change $(P<0.05)$ between the indicated bars

myotubes incubated with GLC/PA than those treated with either nutrient alone (Fig. 1e) It is, therefore, plausible that the observed perturbations in mitochondrial homeostasis seen in this study may, in part, be a consequence of cellular lipotoxicity initiated by the accumulation of molecules such as DAG and ceramide that would be associated with sustained oversupply of fatty acids.

Numerous studies have shown that saturated fatty acids, such as PA, have the capacity to induce IKK-NFkB signalling and can do so via a number of mechanisms including, for example, activation of toll-like receptors [42], MAP kinases (JNK and ERK) [25], excessive ROS generation [43] as well as by enhanced secretion of inflammatory cytokines (e.g., TNF $\alpha$, IL-1 $\beta$, and IL-6) that act via their respective cell surface receptors [44]. Whilst evidence exists associating these inflammatory cytokines with impaired mitochondrial function in some cell types [45, 46], studies linking classical NFkB signalling to mitochondrial dysfunction in 
muscle cells in response to fuel over loading are extremely sparse. However, one potential mechanism by which NFkB may modulate myocellular energy metabolism is via its wellestablished role as a regulator of gene expression [47]. Notable NFkB gene targets include those encoding mitochondrial proteins or transcription factors that regulate expression of key mitochondrial genes. For example, recent work in human glioblastoma cells has highlighted that activated NFkB can bind to two response elements within the gene promoter of the ADP/ATP translocase, ANT-1, resulting in its reduced expression and concomitant decline in mitochondrial ATP production [48]. NFkB activation also represses the activity of PGC1 $\alpha$ [49], which functions as a critical regulator of not only mitochondrial biogenesis/respiration but expression of nuclear respiratory factors (NRFs) that, in turn, modulate expression of mitochondrial proteins such as UCP3 and SDHA [50-52]. An increase in mitochondrial biogenesis/content may serve as an initial response to nutrient excess, but such compensation typically fails in the face of sustained nutrient oversupply. This compensation process appears to be very short lived in our in vitro myotube cultures as whilst we observe an initial increase in the gene expression of PGC1 $\alpha$, SDHA, ANT-1, and UCP3 (during the first $4 \mathrm{~h}$ of nutrient oversupply), we find that this rapidly declines over the succeeding $20 \mathrm{~h}$ myotube incubation period with GLC and PA (Supplementary Fig. S7). This decline correlates with an associated increase in NFkB activation as judged by the loss in IkB $\alpha$ and increased expression of IL- 6 that is observed from $4 \mathrm{~h}$ onwards in myotubes (Supplementary Fig. S7). The notion that these events are likely to be linked is supported by our finding that the targeted inhibition of the IKK-NFkB axis helps mitigate the loss of these mitochondrial proteins and the associated decline in respiratory function. Assessing whether the changes in PGC1 $\alpha$, SDHA, ANT-1 and UCP3 that we see in myotubes in response to fuel overloading are primarily a consequence of a reduction in gene expression or also involve post-transcriptional modulation of processes such as protein synthesis and degradation represent important investigative goals of future work.

Another critical regulator of mitochondrial metabolism and cellular responsiveness to insulin is ROS [53]. Mitochondria are major sources of ROS and generated as a consequence of electron "slippage" within the electron transport chain (ETC) to oxygen during OXPHOS [54]. ROS generated by the ETC are considered important for preserving the normal biological functionality of the respiratory chain. However, under certain circumstances, including mitochondrial substrate overload, sustained and elevated generation of ROS supersedes the capacity of anti-oxidant defence mechanisms leading to increased oxidative stress. Components of the respiratory chain are highly susceptible to oxidative damage and this not only reduces the fidelity and operational efficiency of the ETC, but also results in further augmentation in ROS generation that effectively establishes a viscous cycle driving greater mitochondrial dysfunction [55]. Our findings reveal that heightened ROS production is indeed a feature of mitochondrial substrate overload (Fig. 5) and that this occurs despite an attendant increase in the expression of SOD2, GPX1 and catalase (Supplementary Fig. S2) that most likely represent a cellular stress response designed to limit oxidative stress. Intriguingly, whilst exposing GLC/PA-overloaded myotubes to mitochondrial targeted anti-oxidants such as Mitotempo restrains superoxide accumulation (Supplementary Fig. S1) and confers protection against mitochondrial fragmentation (Supplementary Fig. S4), treatment of myotubes with either Mitotempo or MitoQ could not rescue the decline in mitochondrial respiratory capacity induced by fuel overloading. This observation is most likely explained by the fact that neither anti-oxidant was able to mitigate activation of the IKK-NFkB pathway that we believe initiates the loss of key mitochondrial proteins, such as SDHA, UCP3, and ANT-1 in fuel-overloaded myotubes (Supplementary Fig. S4). SDHA is a critical component within both the TCA cycle and ETC, and consequently, its loss will impact negatively upon both mitochondrial processes. Whilst the precise functional role of UCP3 remains uncertain, its expression in cultured myotubes and rodent skeletal muscle has been linked to modulation of fatty acid oxidation [56-58], mitochondrial integrity, and to management of ROS/oxidative stress [59-62]. Likewise, in addition to catalysing the exchange of cytoplasmic ADP for mitochondrial ATP, ANT-1 can mediate fatty acid-induced uncoupling via its ability to transfer fatty acid anions across the inner mitochondrial membrane [63]. Consequently, mild uncoupling mediated by both UCP3 and ANT-1 may be beneficial in protecting mitochondria against the reducing pressure created by transient increases in fuel supply and, by doing so, restraining the excessive generation/accumulation of ROS that may otherwise promote oxidative damage and impair skeletal muscle insulin sensitivity. The fact that suppressing NFkB activation in fuel-overloaded myotubes with BI605906 or 2DG antagonises loss of UCP3 and ANT-1 and that, under these circumstances, we not only see a measurable increase in mitochondrial proton leak, but observe improved insulin sensitivity in L6 myotubes (Fig. 9) and in muscle of obese Zucker rats [17] is fully congruent with this view. Furthermore, it is noteworthy that the notion that mitochondrial expression/activity of ANT-1 may influence insulin sensitivity of muscle cells is supported by studies in cultured $\mathrm{C} 2 \mathrm{C} 12$ myotubes, in which partial silencing of ANT-1 results in reduced cellular sensitivity to fatty acidinduced uncoupling and a significant reduction in insulinstimulated glucose uptake [63].

In skeletal muscle, reduced mitochondrial respiration has also been linked to aberrant control of mitochondrial dynamics [64-67]. Increased exposure of myotubes to PA 
and fatty acid derivatives such as ceramide promote a low mitochondrial fusion to fission ratio resulting in fragmented, discontinuous mitochondria that not only exhibit diminished capacity for respiration and ATP synthesis, but also is thought to be causally linked to impaired insulin sensitivity and metabolic function [66-68]. Consistent with these observations, GLC/PA overloading of L6 myotubes in our studies induced a profound morphological change in mitochondria from an elongated/tubular network to one that is highly fragmented, indicative of a dynamic shift towards greater fission. The increase in Drp1 (a profission protein) and reduction in MFN2 (a profusion protein) that we see within a mitochondrial-enriched membrane fraction is fully consistent with this view. Strikingly, pharmacological inhibition of the IKK-NFkB axis in GLC/PA-overloaded myotubes not only suppresses the relative changes in Drp1 and MFN2, but reduces fragmentation of the mitochondrial network, in which, significantly, we find was also associated with amelioration in cellular respiratory capacity and insulin action. Since Mitotempo is able to partially restore the tubular mitochondrial network, but not the loss in respiratory capacity in fuel-overloaded myotubes (Supplementary Fig. S4), we postulate that increased fission is likely to be secondary to the increased production of ROS that stems from mitochondrial dysfunction induced by activation of the NFkB pathway. It is also noteworthy that whilst our studies indicate that the activation of canonical NFkB signalling initiated in response to fuel overloading promotes mitochondrial fragmentation, this process can also be influenced by non-canonical NFkB signalling, which is independent of IKK $\beta$ and IKK $\gamma$ (NEMO) but dependent on IKK $\alpha$ dimers. Although we saw no differences in OPA-1 expression in our mitochondrial-enriched membrane fractions from fuel-overloaded L6 myotubes, MEF cells lacking IKK $\alpha$ (but not IKK $\beta$ ) exhibit reduced cellular expression of OPA-1 and display enhanced mitochondrial fission [69]. It is also noteworthy that the activation of the classical NFkB pathway in $\mathrm{C} 2 \mathrm{C} 12$ myotubes can result in suppressed IKK $\alpha$ expression and that this has implications for expression of OXPHOS genes [70]. Regardless of the stimulus promoting a shift towards greater fission, whilst some of the fragmented mitochondria may retain their functional capacity, the structural integrity of others is likely to be compromised (as reflected by an overall reduction in cellular respiratory capacity) and, if not recoverable, will be targeted for clearance by mitophagy. Consistent with this view, we observed a significant increase in the number of mitophagic particles in myotubes subjected to GLC/PA loading, and in line with the protective effects, we suggest that the inhibition of canonical NFkB signalling confers upon mitochondrial integrity and function; this increase in mitophagy was blunted in myotubes that had been cotreated with BI606906. It is worth stating that the observed increase in mitophagy and the associated loss of key mitochondrial proteins (SDHA, ANT1 , and UCP3) that we see in fuel-overloadeded myotubes occur despite their being no significant change in mitochondrial DNA or citrate synthase activity. One potential explanation for this apparent paradox is that whilst our mitophagy reporter assay is highly sensitive and the loss in mitochondrial proteins may be accounted for by a reduction in the transcription and/or translation of their respective genes and products, analysis of mitochondrial DNA may also capture that present within mitochondria during the early stages of the mitophagic process. If so, this would lessen any potential differences in mitochondrial DNA between the treatments that we have performed.

In conclusion, our findings indicate that muscle cells subjected to sustained oversupply of metabolic fuel exhibit an increase in proinflammatory NFkB signalling that is mechanistically linked to diminished mitochondrial function as evidenced by our ability to antagonise the marked decline in respiratory capacity, expression of key mitochondrial maker proteins and the increased fission and mitophagy by pharmacological or genetic repression of the IKK-NFkB axis. Whilst very recent work has shown that sustained inhibition of NFkB can adversely affect muscle development and mitochondrial function during early life its repression has no detrimental effect in adult muscle [71]. Consequently, our observations would imply that therapeutic strategies that help restrain NFkB activation as seen during circumstances of energy excess, such as obesity and age-onset Type II diabetes, may help counter disturbances in mitochondrial homeostasis and impart beneficial effects upon skeletal muscle insulin sensitivity.

Acknowledgements This work was supported by Diabetes UK, BBSRC and MRC. D.S.S is a Arthur and Sadie Pethybridge Diabetes UK Scholar. We are grateful for the technical support offered by Paul Appleton and Graham Ball with the confocal microscope and the Volocity software for analysis of mitophagy as well as members of the Ganley Lab and Dr Chris Lipina for useful discussions.

\section{Compliance with ethical standards}

Conflict of interest The authors declare that they have no conflict of interest.

Open Access This article is distributed under the terms of the Creative Commons Attribution 4.0 International License (http://creativeco mmons.org/licenses/by/4.0/), which permits unrestricted use, distribution, and reproduction in any medium, provided you give appropriate credit to the original author(s) and the source, provide a link to the Creative Commons license, and indicate if changes were made.

\section{References}

1. Feve B, Bastard JP (2009) The role of interleukins in insulin resistance and type 2 diabetes mellitus. Nat Rev Endocrinol 5:305-311 
2. Funaki M (2009) Saturated fatty acids and insulin resistance. J Med Invest 56:88-92

3. Jove $\mathrm{M}$ et al (2005) Palmitate-induced interleukin 6 production is mediated by protein kinase $\mathrm{C}$ and nuclear-factor kappaB activation and leads to glucose transporter 4 down-regulation in skeletal muscle cells. Endocrinology 146:3087-3095

4. Weigert $C$ et al (2004) Palmitate, but not unsaturated fatty acids, induces the expression of interleukin- 6 in human myotubes through proteasome-dependent activation of nuclear factor-kappaB. J Biol Chem 279:23942-23952

5. Boden G (2006) Fatty acid-induced inflammation and insulin resistance in skeletal muscle and liver. Curr Diab Rep 6:177-181

6. Powell DJ et al (2004) Intracellular ceramide synthesis and protein kinase Czeta activation play an essential role in palmitateinduced insulin resistance in rat L6 skeletal muscle cells. Biochem J 382:619-629

7. Barma P et al (2009) Lipid induced overexpression of NF-kappaB in skeletal muscle cells is linked to insulin resistance. Biochim Biophys Acta 1792:190-200

8. Kelley DE et al (2002) Dysfunction of mitochondria in human skeletal muscle in type 2 diabetes. Diabetes 51:2944-2950

9. Ritov VB et al (2005) Deficiency of subsarcolemmal mitochondria in obesity and type 2 diabetes. Diabetes 54:8-14

10. Muoio DM (2014) Metabolic inflexibility: when mitochondrial indecision leads to metabolic gridlock. Cell 159:1253-1262

11. Koves TR et al (2008) Mitochondrial overload and incomplete fatty acid oxidation contribute to skeletal muscle insulin resistance. Cell Metab 7:45-56

12. Lipina C, Hundal HS (2011) Sphingolipids: agents provocateurs in the pathogenesis of insulin resistance. Diabetologia 54:1596-1607

13. Holloszy JO (2013) "Deficiency" of mitochondria in muscle does not cause insulin resistance. Diabetes 62:1036-1040

14. Sparks LM et al (2005) A high-fat diet coordinately downregulates genes required for mitochondrial oxidative phosphorylation in skeletal muscle. Diabetes 54:1926-1933

15. Heilbronn LK et al (2007) Markers of mitochondrial biogenesis and metabolism are lower in overweight and obese insulin-resistant subjects. J Clin Endocrinol Metab 92:1467-1473

16. Petersen KF et al (2004) Impaired mitochondrial activity in the insulin-resistant offspring of patients with type 2 diabetes. N Engl J Med 350:664-671

17. Lipina $C$ et al (2013) Mitochondrial substrate availability and its role in lipid-induced insulin resistance and proinflammatory signalling in skeletal muscle. Diabetes 62:3426-3436

18. Goodpaster BH (2013) Mitochondrial deficiency is associated with insulin resistance. Diabetes 62:1032-1035

19. Bonnard $\mathrm{C}$ et al (2008) Mitochondrial dysfunction results from oxidative stress in the skeletal muscle of diet-induced insulinresistant mice. J Clin Invest 118:789-800

20. Yuzefovych L, Wilson G, Rachek L (2010) Different effects of oleate vs. palmitate on mitochondrial function, apoptosis, and insulin signaling in L6 skeletal muscle cells: role of oxidative stress. Am J Physiol Endocrinol Metab 299:E1096-E1105

21. Morgan MJ, Liu ZG (2011) Crosstalk of reactive oxygen species and NF-kappaB signaling. Cell Res 21:103-115

22. Cherry AD, Piantadosi CA (2015) Regulation of mitochondrial biogenesis and its intersection with inflammatory responses. Antioxid Redox Signal 22:965-976

23. Kwon OS et al (2016) Intramyocellular ceramides and skeletal muscle mitochondrial respiration are partially regulated by Tolllike receptor 4 during hindlimb unloading. Am J Physiol Regul Integr Comp Physiol 311:R879-R887

24. Remels AH et al (2010) TNF-alpha impairs regulation of muscle oxidative phenotype: implications for cachexia? FASEB J 24:5052-5062
25. Green CJ et al (2011) Counter-modulation of fatty acid-induced pro-inflammatory nuclear factor kappaB signalling in rat skeletal muscle cells by AMP-activated protein kinase. Biochem $\mathrm{J}$ 435:463-474

26. Coll $\mathrm{T}$ et al (2008) Oleate reverses palmitate-induced insulin resistance and inflammation in skeletal muscle cells. J Biol Chem 283:11107-11116

27. Hajduch E et al (1998) Constitutive activation of protein kinase $\mathrm{B} \alpha(\mathrm{PKB} \alpha)$ by membrane targeting promotes glucose and System A amino acid transport, protein synthesis and GSK3 inactivation in L6 muscle cells. Diabetes 47:1006-1013

28. Stierwalt HD et al (2018) Insulin-stimulated Rac1-GTP binding is not impaired by palmitate treatment in L6 myotubes. Physiol Rep 6:e13956

29. Yoon JH et al (2015) Proteomic analysis of the palmitate-induced myotube secretome reveals involvement of the annexin A1-formyl peptide receptor 2 (FPR2) pathway in insulin resistance. Mol Cell Proteomics 14:882-892

30. Dimopoulos $\mathrm{N}$ et al (2006) Differential effects of palmitate and palmitoleate on insulin action and glucose utilization in rat L6 skeletal muscle cells. Biochem J 399:473-481

31. Macrae K et al (2013) Defining the role of DAG, mitochondrial function and lipid deposition in palmitate-induced proinflammatory signalling and its counter-modulation by palmitoleate. J Lipid Res 54:2366-2378

32. Bradford MM (1976) A rapid and sensitive method for the quantitation of microgram quantities of protein utilizing the principle of protein-dye binding. Anal Biochem 72:248-254

33. Nisr RB, Affourtit C (2016) Palmitate-induced changes in energy demand cause reallocation of ATP supply in rat and human skeletal muscle cells. BBA 1857:1403-1411

34. McWilliams TG et al (2016) mito-QC illuminates mitophagy and mitochondrial architecture in vivo. J Cell Biol 214:333-345

35. Allen GF et al (2013) Loss of iron triggers PINK1/Parkin-independent mitophagy. EMBO Rep 14:1127-1135

36. Clark K et al (2011) Novel cross-talk within the IKK family controls innate immunity. Biochem J 434:93-104

37. Erion DM, Shulman GI (2010) Diacylglycerol-mediated insulin resistance. Nat Med 16:400-402

38. Holland WL et al (2007) Inhibition of ceramide synthesis ameliorates glucocorticoid-, saturated-fat-, and obesity-induced insulin resistance. Cell Metab 5:167-179

39. Coll $\mathrm{T}$ et al (2010) Activation of peroxisome proliferator-activated receptor- $\{$ delta $\}$ by GW501516 prevents fatty acid-induced nuclear factor- $\{$ kappa $\} \mathrm{B}$ activation and insulin resistance in skeletal muscle cells. Endocrinology 151:1560-1569

40. Di PM, Cocco T, Lorusso M (2000) Ceramide interaction with the respiratory chain of heart mitochondria. Biochemistry 39:6660-6668

41. Perreault L et al (2018) Intracellular localization of diacylglycerols and sphingolipids influences insulin sensitivity and mitochondrial function in human skeletal muscle. JCI Insight 3(3):e96805. https://doi.org/10.1172/jci.insight.96805

42. Senn JJ (2006) Toll-like receptor-2 is essential for the development of palmitate-induced insulin resistance in myotubes. J Biol Chem 281:26865-26875

43. Maloney E et al (2009) Activation of NF-kappaB by palmitate in endothelial cells: a key role for NADPH oxidase-derived superoxide in response to TLR4 activation. Arterioscler Thromb Vasc Biol 29:1370-1375

44. Haversen L et al (2009) Induction of proinflammatory cytokines by long-chain saturated fatty acids in human macrophages. Atherosclerosis 202:382-393

45. Yasuhara $\mathrm{R}$ et al (2005) Interleukin-1beta induces death in chondrocyte-like ATDC5 cells through mitochondrial dysfunction 
and energy depletion in a reactive nitrogen and oxygen speciesdependent manner. Biochem J 389:315-323

46. Ji C et al (2011) IL-6 induces lipolysis and mitochondrial dysfunction, but does not affect insulin-mediated glucose transport in 3T3-L1 adipocytes. J Bioenerg Biomembr 43:367-375

47. Gilmore TD, Garbati MR (2011) Inhibition of NF-kappaB signaling as a strategy in disease therapy. Curr Top Microbiol Immunol 349:245-263

48. Zhang C et al (2017) Transcription factor NF-kappa B represses ANT1 transcription and leads to mitochondrial dysfunctions. Sci Rep 7:44708

49. Alvarez-Guardia D et al (2010) The p65 subunit of NF-kappaB binds to PGC-1alpha, linking inflammation and metabolic disturbances in cardiac cells. Cardiovasc Res 87:449-458

50. Austin S, St-Pierre J (2012) PGC1alpha and mitochondrial metabolism-emerging concepts and relevance in ageing and neurodegenerative disorders. J Cell Sci 125:4963-4971

51. Lopez-Bernardo E et al (2015) 4-Hydroxynonenal induces Nrf2mediated UCP3 upregulation in mouse cardiomyocytes. Free Radic Biol Med 88:427-438

52. Au HC, Scheffler IE (1998) Promoter analysis of the human succinate dehydrogenase iron-protein gene-both nuclear respiratory factors NRF-1 and NRF-2 are required. Eur J Biochem 251:164-174

53. Fisher-Wellman KH, Neufer PD (2012) Linking mitochondrial bioenergetics to insulin resistance via redox biology. Trends Endocrinol Metab 23:142-153

54. Bolisetty S, Jaimes EA (2013) Mitochondria and reactive oxygen species: physiology and pathophysiology. Int J Mol Sci 14:6306-6344

55. Phielix E et al (2008) Lower intrinsic ADP-stimulated mitochondrial respiration underlies in vivo mitochondrial dysfunction in muscle of male type 2 diabetic patients. Diabetes 57:2943-2949

56. Costford SR et al (2008) Long-term high-fat feeding induces greater fat storage in mice lacking UCP3. Am J Physiol Endocrinol Metab 295:E1018-E1024

57. Senese $\mathrm{R}$ et al (2011) Uncoupling protein 3 expression levels influence insulin sensitivity, fatty acid oxidation, and related signaling pathways. Pflug Arch 461:153-164

58. MacLellan JD et al (2005) Physiological increases in uncoupling protein 3 augment fatty acid oxidation and decrease reactive oxygen species production without uncoupling respiration in muscle cells. Diabetes 54:2343-2350

59. Sellares J et al (2007) Oxidative stress time course in the rat diaphragm after freezing-thawing cycles. Respir Physiol Neurobiol 155:156-166
60. Jiang $\mathrm{N}$ et al (2009) Upregulation of uncoupling protein-3 in skeletal muscle during exercise: a potential antioxidant function. Free Radic Biol Med 46:138-145

61. Brand MD et al (2002) Oxidative damage and phospholipid fatty acyl composition in skeletal muscle mitochondria from mice underexpressing or overexpressing uncoupling protein 3 . Biochem J 368:597-603

62. Klumpe I et al (2016) Transgenic overexpression of adenine nucleotide translocase 1 protects ischemic hearts against oxidative stress. J Mol Med (Berl) 94:645-653

63. Sparks LM et al (2016) ANT1-mediated fatty acid-induced uncoupling as a target for improving myocellular insulin sensitivity. Diabetologia 59:1030-1039

64. Johnson ML, Robinson MM, Nair KS (2013) Skeletal muscle aging and the mitochondrion. Trends Endocrinol Metab $24: 247-256$

65. Iqbal S et al (2013) Expression of mitochondrial fission and fusion regulatory proteins in skeletal muscle during chronic use and disuse. Muscle Nerve 48:963-970

66. Jheng HF et al (2012) Mitochondrial fission contributes to mitochondrial dysfunction and insulin resistance in skeletal muscle. Mol Cell Biol 32:309-319

67. Smith ME et al (2013) Mitochondrial fission mediates ceramideinduced metabolic disruption in skeletal muscle. Biochem $\mathrm{J}$ 456:427-439

68. Sebastian D et al (2012) Mitofusin 2 (Mfn2) links mitochondrial and endoplasmic reticulum function with insulin signaling and is essential for normal glucose homeostasis. Proc Natl Acad Sci USA 109:5523-5528

69. Laforge $\mathrm{M}$ et al (2016) NF-kappaB pathway controls mitochondrial dynamics. Cell Death Differ 23:89-98

70. Remels AH et al (2014) Activation of alternative NF-kappaB signaling during recovery of disuse-induced loss of muscle oxidative phenotype. Am J Physiol Endocrinol Metab 306:E615-E626

71. Valentine JM et al (2018) NFkappaB regulates muscle development and mitochondrial function. J Gerontol A Biol Sci Med Sci. https://doi.org/10.1093/gerona/gly262

Publisher's Note Springer Nature remains neutral with regard to jurisdictional claims in published maps and institutional affiliations. 